\title{
Mimetic-metric-torsion with induced axial mode and phantom barrier crossing
}

\author{
Sourav Sur ${ }^{\mathrm{a}}$, Ashim Dutta ${ }^{\mathrm{b}}$, Hiyang Ramo Chothe ${ }^{\mathrm{c}}$ \\ Department of Physics and Astrophysics, University of Delhi, New Delhi 110 007, India
}

Received: 30 October 2020 / Accepted: 30 March 2021 / Published online: 10 April 2021

(C) The Author(s) 2021

\begin{abstract}
We extend the basic formalism of mimeticmetric-torsion gravity theory, in a way that the mimetic scalar field can manifest itself geometrically as the source of not just the trace mode of torsion, but its axial (or, pseudo-trace) mode as well. Specifically, we consider the mimetic field to be (i) coupled explicitly to the well-known Holst extension of the Riemann-Cartan action, and (ii) identified with the square of the associated Barbero-Immirzi field, presumably a pseudo-scalar. The conformal symmetry originally prevaling in the theory would still hold, as the associated Cartan transformations do not affect the torsion pseudo-trace, and hence the Holst term. Demanding the theory to preserve the spatial parity symmetry as well, we show a geometric unification of the cosmological dark sector, and the feasibility of a super-accelerating regime in the course of evolution of the universe. From the observational perspective, assuming the cosmological evolution profile to be very close to that for $\Lambda \mathrm{CDM}$, we further illustrate a smooth crossing of the so-called phantom barrier at a low red-shift, albeit with a restricted parametric domain. Subsequently, we determine the extent of the super-acceleration by examining the evolution of the relevant torsion parameters.
\end{abstract}

\section{Introduction}

Emergence of the theory of mimetic gravity [1-3] has marked a fascinating recent advancement in modified gravity researches purporting to a geometric description of the cosmic dark sector constituents, viz. dark energy (DE) and dark matter (DM) [4-18]. Such a theory has attracted a lot of attention not only for providing an exact mimicry of a dust-like cold dark matter (CDM) component in the standard framework of Friedmann-Robertson-Walker (FRW) cosmology,

\footnotetext{
a e-mail: sourav.sur@gmail.com (corresponding author)

b e-mail: ashim1921@gmail.com

c e-mail: rmo.tnm@gmail.com
}

but also for arousing, via simple extensions, the very prospect of the unification of the entire dark sector. In fact, the original Chemseddine-Mukhanov (CM) model [1] of mimetic gravity ${ }^{1}$ have had a plethora of extensions proposed and studied from various perspectives till date [2,3,22-57], with many interesting implications in both cosmology and astrophysics [58-88].

Strictly speaking, the basic CM theory amounts to a scalartensor reformulation of General Relativity (GR), of a specific sort which exploits the diffeomorphism invariance to reparametrize the physical metric $g_{\mu \nu}$ by a fiducial metric $\widehat{g}_{\mu \nu}$ and a scalar field $\phi$, in a way that $g_{\mu \nu}$ remains invariant under a conformal transformation of $\widehat{g}_{\mu \nu}$. The gravitational conformal degree of freedom thus gets encoded by the field $\phi$, known as the 'mimetic field' [1], which ostensibly has no prior relevance to geometry however. It therefore seems legitimate to look for a generalized mimetic scenario in which $\phi$ can manifest itself geometrically, say for instance, as the source of torsion, which is often regarded as important a space-time characteristic as curvature. ${ }^{2}$ Such a generalization essentially implies contemplating on a conceivable way of expanding the purview mimetic gravity to the metriccompatible Riemann-Cartan $\left(U_{4}\right)$ geometry that admits torsion in addition to curvature. An endeavour pertaining to this has resulted in our mimetic-metric-torsion (MMT) gravity formulation in a recent paper (henceforth, 'Paper 1' [191]), with the eventual enunciation of a viable unified cosmological dark sector paradigm. Nevertheless, scope remains for looking into the subtler aspects of the evolving dark sector,

\footnotetext{
${ }^{1}$ See also some relevant precursors [19-21].

2 Refer to the hefty literature on the long history torsion gravity theories (from the pioneering Einstein-Cartan formulation to the plethora of scenarios that emerged from supergravity, string theory, brane-worlds, etc., or the modified tele-parallelism, Poincaré gauge gravity, and so on) [89-153]. See also the literature on the predicted observable effects of torsion, and their extensive searches [154-190].
} 
by suitably extending the MMT theory, which we intend to do in this work.

From the technical point of view, a consistent MMT formulation primarily amounts to a proper ratification of the principle of mimetic gravity in presence of torsion. This specifically implies the isolation of the conformal (scalar) degree of freedom of gravity via not only the parametrization of the physical metric $g_{\mu \nu}$ by a fiducial metric $\widehat{g}_{\mu \nu}$ and the mimetic field $\phi$, but also that of the physical torsion $T_{\mu \nu}^{\alpha}$ by a corresponding fiducial space torsion $\widehat{T}_{\mu \nu}^{\alpha}$ and $\phi$. We have been able to assert this torsion parametrization in Paper 1 by examining carefully the form of the fiducial space Cartan transformations under which, and a conformal transformation of $\widehat{g}_{\mu \nu}$, the physical fields $g_{\mu \nu}$ and $T_{\mu \nu}^{\alpha}$ are to be preserved. Considering next, from certain standpoints, a contact coupling $\beta(\phi)$ of the mimetic field and the torsional part of the $U_{4}$ Lagrangian, it has been shown that the trace mode $\mathcal{T}_{\mu}$ of torsion gets sourced by $\phi$, which thereby manifests itself geometrically [191]. The resulting energy-momentum tensor resembles that of a perfect fluid, ${ }^{3}$ dubbed the 'MMT fluid', which characterizes dust, albeit with a non-zero pressure. The latter is attained by virtue of an effective potential $W(\phi)$, culminating from $\beta(\phi)$ and its first derivative, in an equivalent formulation illustrated in Paper 1. Any desired expansion history of the universe can therefore be reconstructed in the standard FRW cosmological framework, by suitably choosing the form of the 'MMT coupling' function $\beta(\phi)$. However, in most circumstances such choices lack proper physical motivation, and are merely phenomenological. In Paper 1 though, the consideration of a well-known (and well-motivated) coupling $\beta(\phi) \sim \phi^{2}$ has made the potential $W$ identifiable as a cosmological constant $\Lambda$ (modulo a dimensional factor). The ultimate outcome has therefore been an effective $\Lambda \mathrm{CDM}$ evolution in the MMT cosmological setup, even when the torsion strength diminishes to its unobtrusiveness at late times (thus corroborating to its miniscule experimental signature till date [184-190]).

Nevertheless, the entire MMT formalism in Paper 1 is in some sense minimalistic, since it does not reflect on the actual (intriguing) characteristics of torsion in shaping up the cosmological evolution profile. Specifically, the latter has no direct influence of the antisymmetry property of torsion, unless one assigns an external source for the axial (or pseudotrace) mode $\mathcal{A}_{\mu}$ of torsion. Such an external source, supposedly a non-gravitational field, is anyhow not much interesting, since its admittance may not only affect the predictability of the theory (due to the additional degree(s) of freedom), but also imply the redundancy in aspiring for a geometric unification of the cosmological dark sector. It is imperative therefore

\footnotetext{
${ }^{3}$ Or an imperfect fluid, if one also considers incorporating term(s) containing higher order derivatives of $\phi$, such as $(\square \phi)^{2}$, in the effective Lagrangian [3,59,192-195].
}

to look for extending the basic MMT formalism in such away that apart from torsion's trace $\mathcal{T}_{\mu}$, its axial mode $\mathcal{A}_{\mu}$ (with no assigned external source) can actively influence the dynamical solutions of equations of motion in a given setup (that of FRW cosmology for instance). A simple and natural way to do so is to consider the mimetic field's explicit coupling with not only the torsional part of the $U_{4}$ curvature scalar $\widetilde{R}$, but also its Hodge dual. The latter is commonly known as the 'Holst extension' of the $U_{4}$ Lagrangian, describing modified versions of the conventional metric-torsion theories of Einstein-Cartan (EC) type, or more generally, the refined editions of the canonical (Hilbert-Palatini) connection-dynamic gravitational theories [196-206]. In fact, it had been a generalized Hilbert-Palatini formulation of gravity which had accounted for the Holst term in Holst's original paper in 1996 [197]. Nevertheless, the equivalent term, viz. the Hodge dual of $\widetilde{R}$, in the Einstein-Hilbert formulation had long been known for [196]. In either formulation though, the Holst extension being merely topological (at least, at the minimal level), does not affect the classical dynamics. However, apart from a potential significance in canonical quantum gravity (particularly, relating to Ashtekar-Barbero formulation) [198-201,206-211], the Holst term's presence may reveal intriguingly via gravitational parity violation, possible consequences of which have been studied in various contexts, such as that of an axial torsion induced by the string theoretic Kalb-Ramond field [212-217].

A majority of recent works on the formulations of metrictorsion or connection-dynamic theories have pondered on relaxing the topological characterization of the Holst extension, in which case it can in principle affect the classical dynamics. An appropriate methodology, which has been motivated from various standpoints (such as the chiral anomaly cancellation), is to promote the associated coupling parameter $\gamma$, known as the Berbero-Immirzi (BI) parameter, to the status of a scalar or a pseudo-scalar field [211,217222]. The latter of course is the most suitable option if the theory is to preserve spatial parity symmetry - a consideration amenable to our endeavour on extending the basic MMT formalism by incorporating the Holst term, coupled to the mimetic field $\phi$, in this paper. In fact, a pseudo-scalar (or, an axion-like) BI field $\gamma$ is reasonable from the point of view of ensuring not only that the Holst term would affect the MMT cosmological solutions irrespective of the $\phi$-coupling, but also that such solutions would conform to the general nonperception of the effect(s) of gravitational parity violation, at least at the background level. ${ }^{4}$ It is also worth mentioning here that the admittance of the Holst term does not disturb

\footnotetext{
${ }^{4}$ Note for e.g. that the recent Planck observations do not even provide a reasonably clear indication of the $E-B$ and $T-B$ cross-correlations in the Cosmic Microwave Background (CMB) polarization power spectra [223].
} 
the overall conformal symmetry of the MMT theory, subject to the torsion parametrization suggested in Paper 1, as the associated Cartan transformations leave the axial mode $\mathcal{A}_{\mu}$ of torsion unaffected [191].

Following are the additional steps we take in our extended MMT formulation:

1. Using a Lagrange multiplier, we make an identification of $\phi$ with $\gamma^{2}$, modulo some constant. This of course is meant for a simplification, not only from the point of view of dealing effectively with just a solitary scalar field $\phi$, but also in letting $\phi$ manifest itself geometrically as the source of both the modes $\mathcal{T}_{\mu}$ and $\mathcal{A}_{\mu}$ of torsion. Moreover, note that for such simplification, alongside the preservation of the gravitational parity symmetry, it suffices to identify $\phi$ with any even function of the pseudo-scalar BI field $\gamma$, the $\gamma^{2}$ being just the simplest possible choice.

2. We retain the overall MMT coupling $\beta(\phi)$, but now between $\phi$ and the entire torsional part of the extended $U_{4}$ Lagrangian (the Holst term inclusive). This is essential in the sense that we require to get an equation of motion leading to $\mathcal{T}_{\mu} \propto \partial_{\mu} \phi$ (as in Paper 1), so that the MMT fluid continues to be dust-like ${ }^{5}$ even in the extended setup. Furthermore, we consider the same form of the coupling function, viz. $\beta(\phi) \sim \phi^{2}$, as in Paper 1, for the motivations cited therein. In fact, such a quadratic coupling can have a natural appearance in MMT gravity, as demonstrated in our subsequent paper [229].

3. We also consider augmenting the effective MMT action with a higher derivative $(\square \phi)^{2}$ term, which leads to a nonzero sound speed $c_{s}$ of mimetic matter density perturbations, side-by-side preserving the form of the background cosmological solution one obtains in its absence $[2,3]$.

With this setup, we derive the MMT field equations and constraints, working out in due course the form of the effective potential $W(\phi)$ in Sect. 2 .

Subsequently, in Sect. 3 we carry out the study of the qualitative aspects of the scenario that emerges in the realm of the standard FRW cosmology. In particular, we show that the equation of state parameter $\mathrm{w}_{X}$ of the effective DE component, construed in such a scenario, always tends to fall below the $\Lambda \mathrm{CDM}$ value -1 , thereby exhibiting a superaccelerating phase of cosmic expansion. The transition from $\mathrm{w}_{X}>-1$ to $\mathrm{w}_{X}<-1$, or the so-called phantom barrier crossing, is nonetheless an intriguing pathology, with a fair amount of observational support. For instance, the results of

\footnotetext{
5 That is, the fluid velocity $u_{\mu}$ (which is taken to be equal to $\partial_{\mu} \phi$, in analogy with k-essence cosmologies [224-228]) has to be tangential to the time-like affine geodesics (or the auto-parallels) in the $U_{4}$ spacetime. More specifically, the fluid acceleration must vanish, and thereby confirm the auto-parallel equation (see the Appendix of Paper 1 [191]).
}

the Planck 2018 combined analysis [230] do conform to a certain extent the pre-existing signature of such a crossing at an epoch $t_{C}$ in the recent past phase of evolution of the universe, and that the super-accelerated expansion is still continuing (albeit mildly) at the present epoch $t_{0}$. However, commonly known scalar field DE models (quintessence, k-essence, etc.) cannot usually account for this without the involvement of ghost (or phantom) degree(s) of freedom. There is no such concern though, with our MMT extension scheme outlined above, as the corresponding Lagrangian we propose does not ostensibly consist of any ghost-like term, and in a reduced form looks precisely the same as that in the existing literature on mimetic gravity $[2,3]$. It is the specific form of the potential $W(\phi)$ we get as a consequence of our weird constraining of the system, that leads to the phantom crossing. Actually, the total (mimetic + external) energy-momentum content of the system does not violate any of the energy conditions, except the strong one. It is the mathematical artifact of $W(\phi)$ we interpret as the effective DE constituent in analogy with conventional multi-component cosmological models, that behaves as a phantom. Such an interpretation, although not meant for pinpointing any physical DE candidate, helps us immensely in a bit by bit understanding of the emerging cosmological scenario.

In Sect. 4, we demonstrate the physical realizability of the phantom crossing, i.e. its occurrence at an epoch $t_{C}$ close to but earlier than $t_{0}$. However, this requires our MMT model parametric values to be kept within very narrow domains, which we illustrate after explicitly working out $\mathrm{w}_{X}(t)$, albeit for an approximation justifiable from the point of view of the observational concordance on $\Lambda \mathrm{CDM}$. Specifically, we presume the $\phi$-coupled Holst term is not capable of producing much distortion of the $\Lambda \mathrm{CDM}$ solution we have had in its absence in Paper 1. We determine the validity of the approximation (and subsequently the extent of the superacceleration) in Sect. 5 , by examining the evolution profiles of the torsion parameters, viz. the norms of $\mathcal{T}_{\mu}$ and $\mathcal{A}_{\mu}$, for specific parametric choices.

We conclude in Sect. 6 with a summary and a discussion on some relevant areas open for future investigations, and thereafter demonstrate in the Appendix, the stringent problem of the Ostrogradsky instabilities due to the higher derivative(s), and the possible avoidance of the same, via an alternative Lagrangian extension.

As to the notation and conventions, we adopt those in Paper 1, except using for brevity the units in which the gravitational coupling factor $\kappa^{2} \equiv 8 \pi G_{N}=1$.

\section{General formalism}

To begin with, let us refer the reader to the main tenets of Paper 1 - in particular, the sections 2 and 3 therein, starting with the definition of the torsion tensor 
$T_{\mu \nu}^{\alpha}:=\widetilde{\Gamma}_{\mu \nu}^{\alpha}-\widetilde{\Gamma}_{\nu \mu}^{\alpha}$,

in four-dimensional Riemann-Cartan $\left(U_{4}\right)$ space-time, characterized by an asymmetric (but metric compatible) affine connection $\widetilde{\Gamma}_{\mu \nu}^{\alpha}$. We look to formulate an extended MMT theory in which the (dimensionless) mimetic scalar field $\phi$ can drive the classical dynamics, while inducing both the vector modes of torsion, viz. its trace $\mathcal{T}_{\mu}:=T_{\mu \nu}^{\nu}$ and pseudotrace $\mathcal{A}^{\sigma}:=\epsilon^{\alpha \beta \gamma \sigma} T_{\alpha \beta \gamma}$. For this purpose we resort to the following minimal level generalization of the basic MMT action (of Paper 1):

$$
\begin{aligned}
S= & S^{(m)}+\frac{1}{2} \int d^{4} x \sqrt{-g}\left[R\left(g_{\mu \nu}\right)+\alpha(\square \phi)^{2}\right. \\
& +\beta(\phi)\left\{\Theta\left(g_{\mu \nu}, T_{\mu \nu}^{\alpha}\right)+\frac{1}{2 \gamma} * \Theta\left(g_{\mu \nu}, T_{\mu \nu}^{\alpha}\right)\right\} \\
& \left.+\lambda \cdot(X-1)+v \cdot\left(\phi-s \gamma^{2}\right)\right],
\end{aligned}
$$

where $\Theta$ and ${ }^{*} \Theta$ denote the torsion-dependent part of the $U_{4}$ curvature scalar $\widetilde{R}$ and the Hodge dual of the $U_{4}$ curvature tensor $\widetilde{R}_{\alpha \beta \gamma \delta}$, respectively. In explicit forms, they are expressed in terms of $\mathcal{T}_{\mu}, \mathcal{A}_{\mu}$, and the (pseudo)tracefree mode $\mathcal{Q}_{\mu \nu}^{\alpha}$ of torsion, as

$$
\begin{aligned}
\Theta\left(g_{\mu \nu}, T_{\mu \nu}^{\alpha}\right):= & \widetilde{R}\left(g_{\mu \nu}, T_{\mu \nu}^{\alpha}\right)-R\left(g_{\mu \nu}\right) \\
= & -2 \nabla_{\mu} \mathcal{T}^{\mu}-\frac{2}{3} \mathcal{T}_{\mu} \mathcal{T}^{\mu}+\frac{1}{24} \mathcal{A}_{\mu} \mathcal{A}^{\mu} \\
& +\frac{1}{2} \mathcal{Q}_{\alpha \mu \nu} \mathcal{Q}^{\alpha \mu \nu}, \\
{ }^{\star} \Theta\left(g_{\mu \nu}, T_{\mu \nu}^{\alpha}\right):= & \epsilon^{\alpha \beta \gamma \delta} \widetilde{R}_{\alpha \beta \gamma \delta}\left(g_{\mu \nu}, T_{\mu \nu}^{\alpha}\right) \\
= & -\nabla_{\mu} \mathcal{A}^{\mu}-\frac{2}{3} \mathcal{T}_{\mu} \mathcal{A}^{\mu}+\frac{1}{2} \epsilon^{\alpha \beta \gamma \delta} \mathcal{Q}_{\alpha \beta}^{\mu} \mathcal{Q}_{\mu \gamma \delta},
\end{aligned}
$$

with $R$ being the usual Riemannian $\left(R_{4}\right)$ curvature scalar, and the tensor indices being raised or lowered using the physical metric $g_{\mu \nu}$.

The other constituent terms and symbols in Eq. (2.2) are as explained as follows:

- $S^{(m)}$ is the external matter action.

- $\square \equiv g^{\mu \nu} \nabla_{\mu} \nabla_{\nu}$, with $\nabla_{\mu}$ denoting the $R_{4}$ covariant derivative.

- $\alpha$ is a dimensionless constant parameter measuring the strength of coupling of $(\square \phi)^{2}$.

- $\beta(\phi)$ denotes the overall contact coupling of $\phi$ with $\Theta$, and its Holst extension, viz. $\frac{{ }^{*} \Theta}{2 \gamma}$, with $\gamma$ being the (dimensionless) Berbero-Immirzi (BI) field, which is taken to be a pseudo-scalar (or an axion) in order that the intrinsic parity in the theory is preserved. ${ }^{6}$

$-\lambda$ and $v$ are scalar Lagrange multiplier fields, used respectively for enforcing the mimetic constraint ${ }^{7}$ :

$X \equiv-g^{\mu \nu} \partial_{\mu} \phi \partial_{\nu} \phi=1$

and for identifying $\phi$ with the squared BI field, i.e.

$\phi \equiv s \gamma^{2}$

where $s$ is a dimensionless coupling constant.

Note also the following:

1. The above action (2.2) has merits in itself from the point of view that it comprises of curvature and torsion constructs of only the lowest orders (linear and quadratic respectively). So there is apparently no scale dependence in the theory.

2. The overall conformal symmetry of the theory could be retained even in presence of the $\phi$-coupled Holst term, viz. $\frac{\beta(\phi)}{2 \gamma}{ }^{\star} \Theta \sqrt{-g}$, in the Lagrangian. In principle, just as in Paper 1, we may resort to the physical metric and torsion parametrizations:

$$
\begin{aligned}
g_{\mu \nu} & =\widehat{X} \widehat{g}_{\mu \nu} \\
T_{\mu \nu}^{\alpha} & =\widehat{T}_{\mu \nu}^{\alpha}+q \delta_{[\mu}^{\alpha} \partial_{\nu]}(\ln \widehat{X}),
\end{aligned}
$$

where $\widehat{X}=-\widehat{g}^{\mu \nu} \partial_{\mu} \phi \partial_{\nu} \phi$ and $q$ is a real numerical parameter, which although arbitrary in general, had been set to unity from certain standpoints in Paper 1 (see the section 2 therein [191]). Under the conformal and Cartan transformations of the fiducial metric and torsion, viz.

$$
\begin{aligned}
\widehat{g}_{\mu \nu} & \rightarrow e^{2 \sigma} \widehat{g}_{\mu \nu}, \\
\widehat{T}_{\mu \nu}^{\alpha} & \rightarrow \widehat{T}_{\mu \nu}^{\alpha}+q\left(\delta_{\mu}^{\alpha} \partial_{\nu} \sigma-\delta_{\nu}^{\alpha} \partial_{\mu} \sigma\right),
\end{aligned}
$$

$g_{\mu \nu}$ and $T_{\mu \nu}^{\alpha}$ remain invariant, for any given real scalar function of coordinates $\sigma$. Now, the conformal covariance of the physical torsion (i.e. its conformal invariance in the mixed form $T_{\mu \nu}^{\alpha}$ ) implies the same for its irreducible modes as well. In particular, the transformations (2.9) and (2.10) leave invariant such modes in the respective forms $\mathcal{T}_{\mu}, \mathcal{A}_{\mu}$ and $\mathcal{Q}_{\mu \nu}^{\alpha}$. Moreover, a given conformal

\footnotetext{
${ }^{6}$ In particular, following the convention of Rovelli et al. [199, 200,206], we keep $\gamma$ in the denominator of the coefficient of ${ }^{\star} \Theta$. The factor of 2 , also appearing in the denominator, is just for the purpose of the eliminating certain cumbersome numerical factors in our subsequent derivations.

7 Or, the condition one gets by demanding the physical metric $g_{\mu \nu}$ to be non-singular, and hence invertible.
} 
transformation would not affect the scalar field $\phi$, as it is dimensionless. ${ }^{8}$ By the same token, a conformal transformation would leave the pseudo-scalar $\gamma$ invariant, since $\gamma$ is dimensionless as well. Therefore, expressing the $\phi$ coupled Holst term explicitly as

$$
\begin{aligned}
& \frac{\beta(\phi)}{2 \gamma}{ }^{\star} \Theta \sqrt{-g}=-\frac{\beta(\phi)}{2 \gamma}\left[\partial_{\mu}\left(\sqrt{-g} g^{\mu \nu} \mathcal{A}_{\nu}\right)\right. \\
& \left.+\sqrt{-g}\left\{\frac{2}{3} g^{\mu \nu} \mathcal{T}_{\mu} \mathcal{A}_{\nu}-\frac{1}{2} \epsilon^{\alpha \beta \gamma \delta} g_{\mu \nu} \mathcal{Q}^{\mu \beta} \mathcal{Q}^{\nu}{ }_{\gamma \delta}\right\}\right],
\end{aligned}
$$

we can see its invariance under (2.9) and (2.10), since all of its elements, including the contravariant Levi-Civita tensor density $\varepsilon^{\alpha \beta \gamma \delta} \equiv \sqrt{-g} \epsilon^{\alpha \beta \gamma \delta}$, remain unchanged.

3. The explicit $\phi$-coupling with the Holst term may not seem to be necessary for the MMT extension we are seeking. In fact, it may seem much convenient to consider the action

$$
\begin{aligned}
& S=S^{(m)}+\frac{1}{2} \int d^{4} x \sqrt{-g}\left[R+\alpha(\square \phi)^{2}\right. \\
& \left.\quad+\beta(\phi) \Theta+\frac{1}{2 \gamma} * \Theta+\lambda(X-1)+v\{\phi-f(\gamma)\}\right] .
\end{aligned}
$$

This, unlike (2.2), does not contain any such coupling, and invokes, instead of (2.6), the constraint $\phi=f(\gamma)$, where $f(\gamma)$ needs to be an even function of the axionic BI field $\gamma$, in order to preserve intrinsic parity. We however, prefer to stick to the action (2.2), not only for some technical simplifications, but also for clarity in understanding certain results. Consider for instance the torsion mode $\mathcal{A}_{\mu}$, which is the most crucial ingredient in this extended MMT setup. As shown below (see Eqs. (2.15)), the action (2.2) leads to the result that $\mathcal{A}_{\mu}$ is determined completely by the dynamical solution of the BI field $\gamma$, regardless of the chosen form of the coupling function $\beta(\phi)$. On the other hand, it can be easily verified that $\mathcal{A}_{\mu}$ derived from the action (2.12) would depend not only on $\gamma$ (and $\partial_{\mu} \gamma$ ), but also on $\beta(\phi)$. With either of the actions though, the determination of $\mathcal{A}_{\mu}$ would ultimately require the solution for $\phi$, once the latter is pre-assigned to be identified with some even function of $\gamma$ (for e.g. $\gamma^{2}$ ). Nevertheless, the clarity of wherefrom a given observable (such as the norm of $\mathcal{A}_{\mu}$ ) emerges is somewhat obscured if one uses (2.12).

\footnotetext{
${ }^{8}$ Otherwise, it would be $\phi \rightarrow e^{-\sigma} \phi$, if $\phi$ happens to be a unit mass dimension scalar field, as in most theories [231].
}

Eliminating surface terms, we have the action (2.2) expressed in the form

$$
\begin{aligned}
S= & S^{(m)}+\frac{1}{2} \int d^{4} x \sqrt{-g}\left[R\left(g_{\mu \nu}\right)\right. \\
& +\alpha(\square \phi)^{2}+\lambda(X-1)+v\left(\phi-s \gamma^{2}\right) \\
& +\beta(\phi)\left\{2(\ln \beta)_{\phi} \mathcal{T}_{\mu} \partial^{\mu} \phi-\frac{2}{3} \mathcal{T}_{\mu} \mathcal{T}^{\mu}\right. \\
& \left.+\frac{1}{24} \mathcal{A}_{\mu} \mathcal{A}^{\mu}+\frac{1}{2} \mathcal{Q}_{\alpha \mu \nu} \mathcal{Q}^{\alpha \mu \nu}\right\} \\
& +\frac{\beta(\phi)}{2 \gamma}\left\{\left[(\ln \beta)_{\phi} \partial_{\mu} \phi-\partial_{\mu}(\ln \gamma)\right] \mathcal{A}^{\mu}\right. \\
& \left.\left.-\frac{2}{3} \mathcal{T}_{\mu} \mathcal{A}^{\mu}+\frac{1}{2} \epsilon^{\alpha \beta \gamma \delta} \mathcal{Q}_{\alpha \beta}^{\mu} \mathcal{Q}_{\mu \gamma \delta}\right\}\right],
\end{aligned}
$$

where $(\ln \beta)_{\phi} \equiv \frac{d}{d \phi}(\ln \beta)$, and the indices have been raised and lowered using the physical metric $g_{\mu \nu}$.

Assuming that no external sources of the torsion modes $\mathcal{T}_{\mu}, \mathcal{A}_{\mu}$ and $\mathcal{Q}_{\mu \nu}^{\alpha}$ exist in the matter action $S^{(m)}$, the variation of (2.13) with respect to the each of them leads us to the constraints

$$
\begin{aligned}
\mathcal{T}_{\mu} & =\frac{3}{2}\left[(\ln \beta)_{\phi} \partial_{\mu} \phi-\frac{\partial_{\mu} \gamma}{\gamma\left(\gamma^{2}+1\right)}\right], \\
\mathcal{A}_{\mu} & =\frac{6 \partial_{\mu} \gamma}{\gamma^{2}+1}, \\
\mathcal{Q}_{\mu \nu}^{\alpha} & =0 \quad(\forall \alpha, \mu, \nu),
\end{aligned}
$$

showing that $\mathcal{A}_{\mu}$ is fully dependent on the dynamics of the BI field $\gamma$, whereas $\mathcal{T}_{\mu}$ is not so. Ultimately of course, the identification $s \gamma^{2}=\phi$ [cf. Eq. (2.6)] would leave both these modes, $\mathcal{T}_{\mu}$ and $\mathcal{A}_{\mu}$, as functions of $\phi$ and $\partial_{\mu} \phi$ only. In fact, we may justify such an identification from the point of view of what it entails, viz. the condition $\mathcal{T}_{\mu} \propto \partial_{\mu} \phi$ necessary for the MMT fluid to remain dust-like even in presence of torsion (as proved explicitly in the Appendix of Paper 1 [191]). Accordingly, the norms of $\mathcal{T}_{\mu}$ and $\mathcal{A}_{\mu}$ can finally be expressed as

$$
\begin{aligned}
\mathcal{T}^{2} & \equiv-g^{\mu \nu} \mathcal{T}_{\mu} \mathcal{T}_{\nu}=\frac{9 X}{4 \phi^{2}}\left[\phi(\ln \beta)_{\phi}-\frac{s}{2(\phi+s))}\right]^{2} \\
\mathcal{A}^{2} & \equiv-g^{\mu \nu} \mathcal{A}_{\mu} \mathcal{A}_{v}=\frac{9 s X}{\phi(\phi+s)^{2}}
\end{aligned}
$$

where $X \equiv-g^{\mu \nu} \partial_{\mu} \phi \partial_{\nu} \phi$.

Furthermore, choosing the same quadratic coupling as in Paper 1 (for the motivations cited therein), viz.

$$
\beta(\phi)=\beta_{0}\left(\frac{\phi}{\phi_{0}}\right)^{2}
$$


with $\phi_{0}, \beta_{0}$ as some constant reference values, we may reduce the action (2.2) to

$$
\begin{aligned}
S= & S^{(m)}+\frac{1}{2} \int d^{4} x \sqrt{-g}\left[R+\alpha(\square \phi)^{2}\right. \\
& +\lambda(X-1)-W(\phi) X] .
\end{aligned}
$$

This is of the same form as in Paper 1, albeit with the effective potential

$W(\phi)=2 \Lambda[1-Y(\phi)]$,

where

$Y(\phi)=\frac{s}{16(\phi+s)} \quad$ and $\quad \Lambda=\frac{3 \beta_{0}}{\phi_{0}}$.

Because of the mimetic constraint $X=1$ [cf. Eq. (2.5)], the gravitational field equations are therefore,

$R_{\mu \nu}-\frac{1}{2} g_{\mu \nu} R=\kappa^{2}\left[T_{\mu \nu}^{(m)}+T_{\mu \nu}^{(d)}\right]$,

where $R_{\mu \nu}$ is the usual $\left(R_{4}\right)$ Ricci tensor, $T_{\mu \nu}^{(m)}$ is the matter energy-momentum tensor and $T_{\mu \nu}^{(d)}$ is its mimetic extension. The latter is given by

$$
\begin{aligned}
T_{\mu \nu}^{(d)}= & {\left[R+T^{(m)}-2 W\right] \partial_{\mu} \phi \partial_{\nu} \phi-\frac{1}{2} g_{\mu \nu} W } \\
& +2 \alpha\left(J_{\mu \nu}+J \partial_{\mu} \phi \partial_{\nu} \phi\right),
\end{aligned}
$$

where $T^{(m)}$ denotes the trace of $T_{\mu \nu}^{(m)}$, and

$$
\begin{aligned}
J_{\mu \nu}:= & \frac{1}{2}\left[\partial_{\mu}(\square \phi) \partial_{\nu} \phi+\partial_{\nu}(\square \phi) \partial_{\mu} \phi\right] \\
& -\frac{1}{2} g_{\mu \nu}\left[\partial_{\sigma}(\square \phi) \partial^{\sigma} \phi+\frac{1}{2}(\square \phi)^{2}\right], \\
J= & g^{\mu \nu} J_{\mu \nu}=-\left[\partial_{\sigma}(\square \phi) \partial^{\sigma} \phi+(\square \phi)^{2}\right] .
\end{aligned}
$$

On the other hand, the variation of the action (2.20) with respect to $\phi$ leads to the field equation:

$$
\begin{aligned}
\nabla_{\mu} & {\left[\left(R+T^{(m)}-2 W\right) \partial^{\mu} \phi\right] } \\
& =\frac{1}{2} W_{\phi}-\alpha\left[\square(\square \phi)+2 \nabla_{\mu}\left(J \partial^{\mu} \phi\right)\right],
\end{aligned}
$$

where

$W_{\phi} \equiv \frac{d W}{d \phi}=-2 \Lambda \frac{d Y}{d \phi}=\frac{32 \Lambda}{s} Y^{2}(\phi)$,

for the form of $Y(\phi)$ given above in Eq. (2.22).

\section{Extended MMT cosmological evolution in the standard setup}

In the standard spatially flat FRW space-time background, the mimetic constraint $X=1$ as usual implies that the mimetic field could be treated as a dimensionally rescaled cosmic clock (viz. $\phi \equiv t$, the co-moving time), ${ }^{9}$ without loss of generality [1]. This obviously means $\phi_{0} \equiv t_{0}$, the present age of the universe. Henceforth, we shall use $t$ only, to refer to the mimetic field, as well as the comoving time coordinate, and always denote the total derivative with respect to $t$ by an overhead dot (e.g. $\dot{Y} \equiv d Y / d t$ ).

\subsection{Extended MMT cosmological equations}

Note first that the vanishing of the (pseudo-)tracefree torsion mode $\mathcal{Q}^{\alpha}{ }_{\mu \nu}$ [cf. Eq. (2.16)] is perfectly consistent with the stringent constraints on the torsion tensor required for the FRW metric structure in the $U_{4}$ space-time [232]. In fact, such constraints also imply that only the temporal components of the other torsion modes $\mathcal{T}_{\mu}$ and $\mathcal{A}_{\mu}$ should exist, and hence by Eqs. (2.14) and (2.15), the scalar fields $\phi$ and $\gamma$ should only depend on time. Secondly, because of the form of $J_{\mu \nu}$ [cf. Eq. (2.25)], that stems out of the $\alpha(\square \phi)^{2}$ term in the action (2.20)), the expression (2.24) for $T_{\mu \nu}^{(d)}$ cannot in general be recast in the form of a perfect fluid energy-momentum tensor. Nevertheless, in the FRW space-time one has the relationship

$\square \phi=-3 H$,

where $H=\frac{\dot{a}}{a}$ is the Hubble parameter, with $a(t)$ denoting the scale factor. Therefore, as is well-known [2,3,192], the Friedmann and Raychaudhuri equations (that could be derived from Eqs. (2.23) and (2.27)) reduce to their standard forms (viz. the ones in presence of a perfect fluid matter) only with the effective potential $W(t)$ rescaled by a constant factor that depends on the coefficient $\alpha$ of the $(\square \phi)^{2}$ term in the mimetic action. Given the form of our MMT potential (2.21), this simply implies a rescaling of the constant parameter $\Lambda$ (which we shall consider as a cosmological constant):

$\Lambda \rightarrow\left(1+3 c_{s}^{2}\right) \Lambda$, with $c_{s}^{2}=\frac{\alpha}{2-3 \alpha}$.

The quantity $c_{s}$ here is nothing but the sound speed of the mimetic field perturbations, which the $\alpha(\square \phi)^{2}$ term in the mimetic action famously leads to $[2,3,192]$.

\footnotetext{
${ }_{9}$ Actually, $\phi \equiv \kappa t$. The dimensional factor $\kappa=\sqrt{8 \pi G_{N}}$ is however set to unity in this paper.
} 
Consider now the cosmological matter to be the (pressureless) baryonic dust, specifically for the study of the late-time evolution of the universe:

$T_{\nu}^{(m) \mu}=\operatorname{diag}\left[-\rho^{(m)}, 0,0,0\right]$,

with the corresponding energy density given by

$\rho^{(m)}(t)=\frac{\rho_{0}^{(m)}}{a^{3}(t)}, \quad$ where $\left.\quad \rho_{0}^{(m)} \equiv \rho^{(m)}\right|_{t=t_{0}}$.

The total effective energy density and pressure, $\rho$ and $p$, which satisfy the standard Friedmann and conservation equations, viz.

$H^{2}=\frac{\rho}{3} \quad$ and $\quad \dot{\rho}=-3 H(\rho+p)$,

are then, respectively,

$\rho=\rho^{(m)}+\rho^{(d)}=R+3 p$,

$p=p^{(d)}=-\frac{1}{2} W(t)=-\Lambda[1-Y(t)]$.

These of course reduce to that for the effective $\Lambda \mathrm{CDM}$ solution in Paper 1, once the BI field is turned off, i.e. in the limit the parameter $s \rightarrow 0$, whence $Y(t) \rightarrow 0$. In general however, (for $s \neq 0$ ) it is reasonable to consider the MMT energy density $\rho^{(d)}$ to consist of a pressureless (dust-like) cold dark matter $(\mathrm{CDM})$ density $\rho^{(c)}$, and a left-over $\rho_{X}$, which may be treated as the effective dark energy (DE) density. In other words, while seeking a MMT cosmological dark sector, we may conveniently decompose its energy density as

$\rho^{(d)}(t)=\rho^{(c)}(t)+\rho_{X}(t)$,

with

$\rho^{(c)}(t)=\frac{\rho_{0}^{(c)}}{a^{3}(t)}, \quad$ where $\left.\rho_{0}^{(c)} \equiv \rho^{(c)}\right|_{t=t_{0}}$.

The effective MMT fluid pressure $p^{(d)}$ (that equals the total pressure $p$ ), on the other hand, may inevitably be attributed to that of the effective DE constituent, i.e.

$p_{X}(t)=p(t)=p^{(d)}(t)=-\Lambda[1-Y(t)]$.

Hence, from Eq. (3.5) it follows that

$\dot{\rho}_{X}(t)=-3 H(t)\left[1+\mathrm{w}_{X}(t)\right] \rho_{X}(t)$,

for a barotropic DE equation of state (EoS):

$\mathrm{w}_{X}(t):=\frac{p_{X}(t)}{\rho_{X}(t)}=-\frac{\Lambda[1-Y(t)]}{\rho_{X}(t)}$.
3.2 Cosmic super-acceleration in the extended MMT scenario

Eqs. (3.11) and (3.12) lead to the first order differential equation

$\dot{\mathrm{w}}_{X}(t):=-\left[\frac{\dot{Y}(t)}{1-Y(t)}-3\left\{1+\mathrm{w}_{X}(t)\right\} H(t)\right] \mathrm{w}_{X}(t)$.

Now, given the form

$Y(t)=\frac{s}{16(t+s)}$,

we have

$$
\begin{aligned}
\dot{Y}(t) & =-\frac{s}{16(t+s)^{2}}=-\frac{16}{s} Y^{2}(t), \\
\frac{1}{1-Y(t)} & =\frac{16(t+s)}{16 t+15 s} .
\end{aligned}
$$

Hence, for the constant parameter $s$ to be presumably positive-valued, ${ }^{10} \dot{Y}$ is negative definite, whereas $(1-Y)^{-1}$ is positive definite. Also, in an expanding universe, the Hubble parameter $H$ is positive definite. Therefore in Eq. (3.13), the first term within the square brackets is negative definite, and so is the second term, as long as $\mathbf{w}_{X}>-1$. Since, in any viable DE model, the corresponding EoS parameter $w_{X}$ is negative-valued, at least from reasonably high red-shifts ( $\sim 100$ or so) all the way to the distant future, we may safely say that $\dot{\mathrm{w}}_{X}<0$ whenever $\mathrm{w}_{X}>-1$. The inferences that can be drawn from this are as follows:

(i) If we have a non-phantom (i.e. accelerating, but not super-accelerating) regime in a moderately distant past, i.e. $w_{X}>-1$, then with the progress of time $\mathrm{w}_{X}$ would decrease and tend to attain the value -1 , as the slope $\dot{\mathrm{W}}_{X}$ is always negative. In fact, at $t=t_{C}$, the epoch at which $\mathrm{w}_{X}$ equals -1 , the slope $\dot{\mathrm{w}}_{X}<0$. Thereafter (i.e. with the advancement of time further beyond $t_{C}$ ), $\dot{\mathrm{w}}_{X}$ would continue to remain negative, albeit with progressively lesser magnitude, at least for a certain period after which it may become positive. Hence, given the circumstances, we would definitely have a crossing of the phantom barrier (or the $\mathrm{w}_{X}=-1$ point) at the epoch $t=t_{C}$. There of course remain issues of sorting out finer details, for e.g. the condition

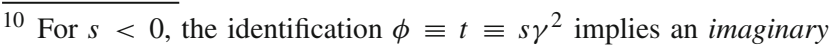
BI field. We prefer to keep that possibility out of the scope of this paper, although it is not unphysical, and in some sense reminiscent of Ashtekar's original works on the canonical quantum gravity formulation [207,208].
} 
for such a crossing to happen in the past (i.e. $t_{C}<t_{0}$ ), the possibility of the phantom regime to be transient (i.e. $\dot{\mathrm{w}}_{X}$ becomes positive after a certain lapse of time beyond $t_{C}$, and increases to the extent that $\mathrm{w}_{X} \geq-1$ once more), and so on. However, addressing to these issues requires us to solve Eq. (3.13) explicitly by setting up the appropriate boundary conditions, or(and) assert somehow the appropriate range of values of the parameter $s$. Of course, appropriateness here implies satisfying the requirement $t_{C}>t_{T}$, where $t_{T}$ denotes the epoch of transition from a decelerating phase to an accelerating phase. Otherwise, our presumption of having a non-phantom regime in the past, and consequently the phantom barrier crossing, would be invalidated.

(ii) If, on the contrary, we have a phantom (or, superaccelerating) regime in a moderately distant past, i.e. $\mathrm{w}_{X}<-1$, then $\dot{\mathrm{w}}_{X}$ would be $<0(>0)$ depending on whether the first term within the square brackets in Eq. (3.13) is bigger (smaller) than the second term therein. Evidently, there would be no phantom barrier crossing for $\dot{\mathrm{w}}_{X}<0$, whereas for $\dot{\mathrm{w}}_{X}>0$ such a crossing can happen, albeit from a phantom phase to a nonphantom phase. In either case though, no realistic cosmological evolution could be extracted unless we have a prior knowledge of a decelerating phase, followed by an accelerating but non-phantom phase, to precede the super-accelerating regime that we assume. This means that our consideration must have to be diverted back to the point (i) above, in order that a viable phantom crossing MMT model can emerge.

The bottomline is therefore that the extended MMT setup we are considering here would definitely give rise to a superaccelerating cosmological scenario, via a fractional reduction in the effective potential $W(t)$ by an amount $Y(t)$, over and above the cosmological constant $\Lambda$ we have had in Paper 1 . Most importantly, this super-acceleration is not apparently stemming out of any ghost (or phantom) degree(s) of freedom in the effective action (2.2). It is the result of the specific inverse time-dependence $Y(t)$ acquires [cf. Eq. (3.14)], due to our chosen coupling $\beta(\phi) \sim \phi^{2}$, of the mimetic field and the Holst term, as well as the identification (2.6). In fact, as we shall explain below, there is nothing unusual with the energy conditions, at least as long as the strength of $Y(t)$, measured by the parameter $s$ in Eq. (3.14), is reasonably weak. After all, it is this parameter $s$ which decides not only the extent of the super-acceleration, but also the physical realizability of the same (see the next section for further clarification). A large value of $s$ compared to the original MMT coupling strength $\beta_{0}\left(=\Lambda t_{0 / 3}\right)$ necessarily means a strong super-acceleration, culminating from a large distortion of the $\Lambda \mathrm{CDM}$ solution, that we had in Paper 1 because of the constant potential $\bar{W}=$
$2 \Lambda$ therein. Since any major deviation from $\Lambda \mathrm{CDM}$ is not even remotely supported by the observations, we may safely assume the parameter $s$ to be small, and for all practical purposes treat $Y(t)$ as a perturbation over $\bar{W}=2 \Lambda$. This may also be reasoned from a purely theoretical standpoint, as follows:

Recall that by Eq. (3.14), the fractional correction $Y(t)$ is a monotonically decreasing function of time. Its maximum value is only $\frac{1}{16}$, and that too is attained at $t=0$. Therefore, at least for the late time cosmology, we may expect $Y(t)$ not to make any strong impact on the $\Lambda \mathrm{CDM}$ solution one gets in its absence. Such an expectation would possibly surmount to the level of a conviction once we consider the smallness of $s$. More specifically, a small $s$ would imply that the solution of Eq. (3.11) may not possibly change its limiting value, viz. $\left.\rho_{X}\right|_{s=0}=\Lambda$, by an amount of the order of $\Lambda$ itself. Hence, $\Lambda$ being the dominant term in it, $\rho_{X}$ would be positive-valued, and so would be the total density $\rho\left(=\rho^{(m)}+\rho^{(c)}+\rho_{X}\right)$. The positivity of $\rho_{X}$ may nonetheless seem a bit surprising, since it implies that our effective DE component is not characteristically phantom-like, yet it leads to a phantom barrier crossing. However, remember that such a component is liable to show some weirdness, as it is merely a mathematical artifact, meant only for facilitating our understanding of the cosmological dynamics in analogy with the conventional scalar field induced DE models.

Now, at least for small $s$, the phantom barrier would not be breached to that extent which would imply that the quantity $\left|1+\mathrm{w}_{X}\right| \sim \mathcal{O}(1)$, i.e. $\left|p_{X}\right|$ exceeding $\rho_{X}$ by an amount of the order of $\rho_{X}$ itself. This can be inferred right away from Eqs. (3.10) and (3.14) which lead to

$\rho_{X}(t)+p_{X}(t)=\delta \rho_{X}(t)+\frac{\Lambda s}{16(t+s)}$,

where $\delta \rho_{X}(t)=\rho_{X}(t)-\Lambda$ denotes the correction in the DE density over its limiting value $\Lambda$. The second term on the right hand side of Eq. (3.17) is positive definite (under our presumption $s>0$ of course). Therefore, $\left(\rho_{X}+p_{X}\right)$ would become negative only when $\delta \rho_{X}$ becomes negative and exceeds that term in magnitude. However, as argued above, $\delta \rho_{X}$ cannot be of the order of $\Lambda$. So there is no question of $\left|\rho_{X}+p_{X}\right|$ reaching up to the order of $\rho_{X}$. By all means then, the total equation of state parameter of the system, $\mathrm{w}=p / \rho$, would not get reduced to a value $<-1$, even in the phantom regime. The reason is obvious - in order to make $w<-1$, i.e. $(\rho+p)<0$, the correction $\left|\delta \rho_{X}\right|$ would need to overcome not only the second term in Eq. (3.17), but also $\rho^{(m)}$ and $\rho^{(c)}$, which is impracticable at least for small $s$. We would thus have $\rho+p>0$, in addition to $\rho>0$. Therefore, both the null and weak energy conditions would hold [233235]. Moreover, w not crossing -1 means that it would have a fractional value, i.e. $|p|<\rho$. So the dominant energy 
condition would hold as well. Nevertheless, the strong energy condition, viz. $\rho+3 p>0$, or $\mathrm{w}>-\frac{1}{3}$, would be violated as usual for an accelerated cosmic expansion.

\section{Viable MMT cosmology with phantom barrier crossing}

Let us, for convenience, treat the inverse squared BI field as an effective coupling function, viz.

$\sigma(\tau):=\gamma^{-2}(\tau)$, where $\tau=\frac{t}{t_{0}}$

Since $\gamma^{-2}=s \phi^{-1} \equiv s t^{-1}$, by Eq. (2.6), we can write

$\sigma(\tau)=\frac{\sigma_{0}}{\tau}, \quad$ where $\left.\quad \sigma_{0} \equiv \sigma\right|_{\tau=1}=\frac{s}{t_{0}}$

Accordingly, Eq. (3.13) can be recast in the form

$\frac{d \mathrm{w}_{X}}{d \tau}:=-\left[\frac{1}{1-Y(\tau)} \frac{d Y}{d \tau}-3 t_{0}\left(1+\mathrm{w}_{X}(\tau)\right) H(\tau)\right] \mathrm{w}_{X}(\tau)$,

where

$Y(\tau)=\frac{\sigma(\tau)}{16[1+\sigma(\tau)]}=\frac{\sigma_{0}}{16\left(\tau+\sigma_{0}\right)}$.

Therefore,

$$
\begin{aligned}
& \frac{d Y}{d \tau}=-\frac{16}{\sigma_{0}} Y^{2}(\tau), \\
& \frac{1}{1-Y(\tau)}=\frac{16\left(\tau+\sigma_{0}\right)}{16 \tau+15 \sigma_{0}},
\end{aligned}
$$

which when substituted back in Eq. (4.3) gives

$$
\begin{aligned}
\frac{d \mathrm{w}_{X}}{d \tau}:= & {\left[\frac{\sigma_{0}}{\left(\tau+\sigma_{0}\right)\left(16 \tau+15 \sigma_{0}\right)}\right.} \\
& \left.+3 t_{0}\left\{1+\mathrm{w}_{X}(\tau)\right\} H(\tau)\right] \mathrm{w}_{X}(\tau) .
\end{aligned}
$$

\subsection{Effective dark energy state parameter in a linear approximation}

In order to solve Eq. (4.7), for the effective dark energy EoS parameter $\mathrm{w}_{X}$, one first requires to find the functional form of $H(\tau)$ by solving the cosmological equations (3.5). While getting an exact analytical solution is always a hard proposition, numerical methods may be applied, with a lot of intuition though, from the point of view of setting priors on the parameters $\sigma_{0}, t_{0}$ and so on. We may however take a shorter course, since for the reasons given earlier, it suffices us to look for small deviations from the $\Lambda \mathrm{CDM}$ scenario. Let us therefore resort to the following expansions:

$$
\begin{aligned}
\mathrm{w}_{X}(\tau) & =\mathrm{w}_{X}^{(0)}(\tau)+\sigma_{0} \mathrm{w}_{X}^{(1)}(\tau)+\sigma_{0}^{2} \mathrm{w}_{X}^{(2)}(\tau)+\cdots, \\
H(\tau) & =H^{(0)}(\tau)+\sigma_{0} H^{(1)}(\tau)+\sigma_{0}^{2} H^{(2)}(\tau)+\cdots,
\end{aligned}
$$

by assuming the parameter $s$ to be small enough, so that $\sigma_{0}=s / t_{0}$ can be treated as a perturbation parameter. The unperturbed quantities correspond to those for $\Lambda \mathrm{CDM}$, viz. ${ }^{11}$

$\mathrm{w}_{X}^{(0)}=-1$ and $H^{(0)}(\tau)=\bar{H}(\tau)$,

whereas the linear order perturbation equation, obtained from Eqs. (4.7)-(4.9), is given by

$\frac{d \mathrm{w}_{X}^{(1)}}{d \tau}=-\left[\frac{1}{16 \tau^{2}}+3 t_{0} \mathrm{w}_{X}^{(1)}(\tau) \bar{H}(\tau)\right]$

For simplicity (and also the adequacy justified later on), we shall confine ourselves to this order of perturbation in what follows.

Consider first the zeroth order scenario, i.e. the effective $\Lambda \mathrm{CDM}$ evolution in the MMT cosmological setup. The total pressure being $\bar{p}=-\Lambda$, the corresponding Friedmann and conservation equations lead to following equation for the total energy density

$\frac{d \bar{\rho}}{d \tau}=-t_{0} \sqrt{3 \bar{\rho}(\tau)}[\bar{\rho}(\tau)-\Lambda]$

with solution

$\bar{\rho}(\tau)=\Lambda \operatorname{coth}^{2} \zeta(\tau)$,

where

$\zeta(\tau)=\zeta_{0} \tau, \quad \zeta_{0}=\left.\zeta\right|_{\tau=1}=\frac{t_{0} \sqrt{3 \Lambda}}{2}$

So, by the $\Lambda \mathrm{CDM}$ Friedmann equation, the corresponding Hubble parameter assumes the form

$\bar{H}(\tau)=\sqrt{\frac{\bar{\rho}(\tau)}{3}}=\frac{2 \zeta_{0}}{3 t_{0}} \operatorname{coth}\left(\zeta_{0} \tau\right)$

Using this in (4.14) we have, more conveniently,

$\zeta(\tau)=\tanh ^{-1} \sqrt{\bar{\Omega}^{(\Lambda)}(\tau)}, \quad \zeta_{0}=\tanh ^{-1} \sqrt{\bar{\Omega}_{0}^{(\Lambda)}}$,

11 Throughout this paper, we denote the $\Lambda \mathrm{CDM}$ parameters and functions by placing an overbar, for e.g. the $\Lambda \mathrm{CDM}$ total energy density denoted by $\bar{\rho}$, the corresponding total pressure denoted by $\bar{p}$, and so on. 
where $\bar{\Omega}^{(\Lambda)}=\Lambda / \bar{\rho}$ is the $\Lambda$-density parameter (specific to the zeroth order case), with value $\bar{\Omega}_{0}^{(\Lambda)}$ at the present epoch $t=t_{0}($ or $\tau=1)$.

Resort now to the linear (or first) order approximation, in which case we can determine

$\mathrm{w}_{X}(\tau) \approx-1+\sigma_{0} \mathbf{w}_{X}^{(1)}(\tau)$,

by plugging Eq. (4.15) back in Eq. (4.11), and solving the resulting equation. This gives

$$
\begin{aligned}
\mathbf{w}_{X}^{(1)}(\tau)= & \frac{1}{16}\left[\frac{1}{\tau}-\left(1-16 \mathbf{w}_{X 0}^{(1)}\right) \frac{\sinh ^{2} \zeta_{0}}{\sinh ^{2}\left(\zeta_{0} \tau\right)}\right. \\
& \left.-\frac{\zeta_{0}\left\{\operatorname{Shi}\left(2 \zeta_{0} \tau\right)-\operatorname{Shi}\left(2 \zeta_{0}\right)\right\}}{\sinh ^{2}\left(\zeta_{0} \tau\right)}\right],
\end{aligned}
$$

where $\left.\mathbf{w}_{X 0}^{(1)} \equiv \mathbf{w}_{X}^{(1)}\right|_{\tau=1}$, and

$\operatorname{Shi}(x):=\int^{x} \frac{\sinh x^{\prime}}{x^{\prime}} d x^{\prime}=\sum_{k=0}^{\infty} \frac{x^{2 k+1}}{(2 k+1)^{2}(2 k) !}$,

is the hyperbolic sine integral function.

In order to get a quantitative measure of the extent to which $\mathrm{w}_{X}$ deviates from the $\Lambda \mathrm{CDM}$ value $\mathrm{w}_{X}^{(0)}=-1$ at different epochs of time, it is convenient for us to figure our the circumstances in which the time-evolution of the percentage change

$$
\begin{aligned}
\mu(\tau) & :=\frac{\mathrm{w}_{X}(\tau)-\mathrm{w}_{X}^{(0)}(\tau)}{\mathrm{w}_{X}^{(0)}(\tau)} \times 100 \\
& =-100\left[1+\mathrm{w}_{X}(\tau)\right],
\end{aligned}
$$

would comply with the observations. From Eqs. (4.17), (4.18) and (4.20) we see that at the present epoch $\tau=1$ ( or $t=t_{0}$ ),

$\sigma_{0} \mathbf{w}_{X 0}^{(1)} \approx-\frac{\mu_{0}}{100}, \quad$ where $\mu_{0}=\mu\left(t_{0}\right)$.

Observational estimations of the value of $\mathrm{w}_{X}\left(t_{0}\right)$, considering say, the well-known Chevallier-Polarski-Linder (CPL) ansatz $[239,240]$, imply that the percentage correction $\mu\left(t_{0}\right) \equiv \mu_{0}$ to be typically $\mathcal{O}(1)$ or lesser, up to the $1 \sigma$ error limits. For instance, the Planck 2018 results of the analysis of CMB TT,TE,EE+lowE combined with Lensing, Baryon Acoustic Oscillation (BAO) and type Ia Supernovae $(\mathrm{SNe})$ data show [230]

$\mathrm{w}_{X}\left(t_{0}\right)=-1.028 \pm 0.032$,

at $68 \%$ confidence level. This means $\mu_{0} \in(-0.04,6)$, which indicates not only a cosmological evolution very close to
$\Lambda \mathrm{CDM}$, but also a tendency of the universe to be in a superaccelerating (or phantom) state at least at low red-shifts. Therefore, while seeking a viable model of a dynamical dark phase of the universe, one requires to ponder heavily on prioritizing the demand that the time-evolution of $\mathrm{w}_{X}$ must turn out to be slow enough, so that the $\Lambda \mathrm{CDM}$ solution is not distorted too much at least at the later epochs. So our linear approximation is duly justified. Moreover, it is desirable to have a provision for a phantom barrier crossing at an epoch in the recent past regime, in order that the expansion of the universe super-accelerates, albeit mildly, at the present epoch. After all, allowing for such a crossing provides flexibility in making statistical estimations of the model parameters (using the observational data), as opposed to say, the quintessence scenarios in which one has to comply with the condition $\mathrm{w}_{X}>-1$ beforehand. As we shall see in the next subsection, our approximated solution for $\mathrm{w}_{X}$ can indeed lead to the perception of a viable phantom crossing scenario, however at the expense of restricting the domain of the parameters $\sigma_{0}$ and $\mu_{0}$ severely.

\subsection{Parametric bounds from observational results}

For illustrative purposes, let us resort to the results of Planck 2018 TT,TE,EE+lowE+Lensing+BAO combined analysis for the base $\Lambda \mathrm{CDM}$ model [230], as in Paper 1. In particular, we shall use the best fit value of the estimated $\Lambda$-density parameter at the present epoch, viz. $\bar{\Omega}_{0}^{(\Lambda)}=0.6889$, which implies

$\zeta_{0}=\tanh ^{-1} \sqrt{\bar{\Omega}_{0}^{(\Lambda)}}=1.1881$.

Now, it is obvious that the linear approximation would not lead to a significant effect on the deceleration-to-acceleration transition epoch $t_{T}$. So to determine the realistic parametric range of $\sigma_{0}$ or $\mu_{0}$ with reasonable precision, it would suffice us to approximate $t_{T}$ to be that for $\Lambda \mathrm{CDM},{ }^{12}$ which can be obtained simply from the condition for cosmic acceleration, viz. $\overline{\mathrm{W}}\left(\tau_{T}\right)=-\frac{1}{3}$, where $\tau_{T} \equiv t_{T} / t_{0}$ and

$\overline{\mathbf{W}}(\tau):=\frac{-\Lambda}{\bar{\rho}(\tau)}=-\bar{\Omega}^{(\Lambda)}(\tau)=-\tanh ^{2}\left(\zeta_{0} \tau\right)$

is the $\Lambda \mathrm{CDM}$ total EoS parameter. With $\zeta_{0}=1.1881$, it follows that

$\tau_{T} \equiv \frac{t_{T}}{t_{0}}=\frac{1}{\zeta_{0}} \tanh ^{-1} \sqrt{\frac{1}{3}}=0.554$.

\footnotetext{
${ }_{12}$ In fact, the deviation of the actual $t_{T}$ from its $\Lambda \mathrm{CDM}$ value turns out to be about $0.056 \sigma_{0}$, as shown rigorously by using the Planck 2018 TT,TE,EE+lowE+Lensing+BAO results in a subsequent paper [236]. With a presumably small $\sigma_{0}$ value, this deviation is really quite insignificant.
} 
Moreover, from the parametric relation (4.21), we see that the solution (4.18) can admit a viable phantom barrier crossing only when, for a fixed $\sigma_{0}$, the range of $\mu_{0}$ is restricted, or vice versa. Of course, by 'viable' we mean that such a crossing has to take place at an epoch $t_{C}$ in the near past (or low redshift) era of the evolving universe, when the latter's expansion is already accelerating, after the end of the decelerating phase at $t=t_{T}$. In other words, $t_{C}$ must lie within the temporal range $\left(t_{T}, t_{0}\right)$.

Again, since the phantom crossing implies

$0=1+\mathrm{w}_{X}\left(\tau_{C}\right) \approx \mathrm{w}_{X}^{(1)}\left(\tau_{C}\right)$, where $\tau_{C} \equiv \frac{t_{C}}{t_{0}}$,

we have from the above Eqs. (4.18) and (4.21),

$$
\begin{aligned}
\mu_{0} \approx & \frac{25 \sigma_{0}}{4} \frac{\zeta_{0}}{\sinh ^{2} \zeta_{0}}\left[\frac{\sinh ^{2}\left(\zeta_{0} \tau_{C}\right)}{\zeta_{0} \tau_{C}}-\frac{\sinh ^{2} \zeta_{0}}{\zeta_{0}}\right. \\
& \left.-\operatorname{Shi}\left(2 \zeta_{0} \tau_{C}\right)+\operatorname{Shi}\left(2 \zeta_{0}\right)\right] .
\end{aligned}
$$

This relationship is crucial for determining the parametric bounds, as we shall see below.

I. For a fixed $\sigma_{0}$, the requisite parametric range of $\mu_{0}$ that meets the criterion $t_{C} \in\left(t_{T}, t_{0}\right)$ is by no means broad, especially given the condition that $\sigma_{0}$ has to be small in order to make the linear approximation tenable. In particular, even if we consider fixing $\sigma_{0}$ at a reasonably significant value 0.05 , the plots of the percentage correction $-\mu(\tau)$ [cf. Eq. (4.20)] in Fig. 1a, for various parametric values of $\mu_{0}$, make it evident that the latter cannot go much further beyond 0.1. Otherwise (for e.g. when $\mu_{0}=0.15$ ), $t_{C}$ would fall below $t_{T}$, thus making the phantom crossing unrealistic. Analytically, this can be easily seen from Eq. (4.27), since with $\zeta_{0}=1.1881$ and $t_{T}=0.554 t_{0} \quad$ [cf. Eqs. (4.23) and (4.25) respectively], the criterion $t_{C} \in\left(t_{T}, t_{0}\right)$ implies

$0<\mu_{0} \lesssim 2.367 \sigma_{0}\left(=0.118\right.$, for $\left.\sigma_{0}=0.05\right)$.

The solid curve in Fig. 1a shows the $-\mu(\tau)$ variation for the optimum value $\mu_{0}=0.118$. The minimum (or turning) point of this curve, at $\tau=\tau_{m}=0.945$, is of particular significance since it marks the earliest possible occurence of the minimum of $-\mu(\tau)$ corresponding to a $\mu_{0}$ value legitimate for a realistic phantom crossing. More specifically, at the crossing point $\tau=\tau_{C}$ the function $-\mu(\tau)$ requires to be decreasing at such a rate that the minimum is reached very close to the present epoch, or afterwards. This is in fact a general requirement, irrespective of the chosen fixed value $\sigma_{0}=0.05$, as verified below.

From Eqs. (4.17) and (4.20) we see that the minimization of $-\mu(\tau)$ implies that of $\mathbf{w}_{X}^{(1)}(\tau)$. Therefore, using Eqs. (4.11), (4.15), (4.18) and (4.21), we get

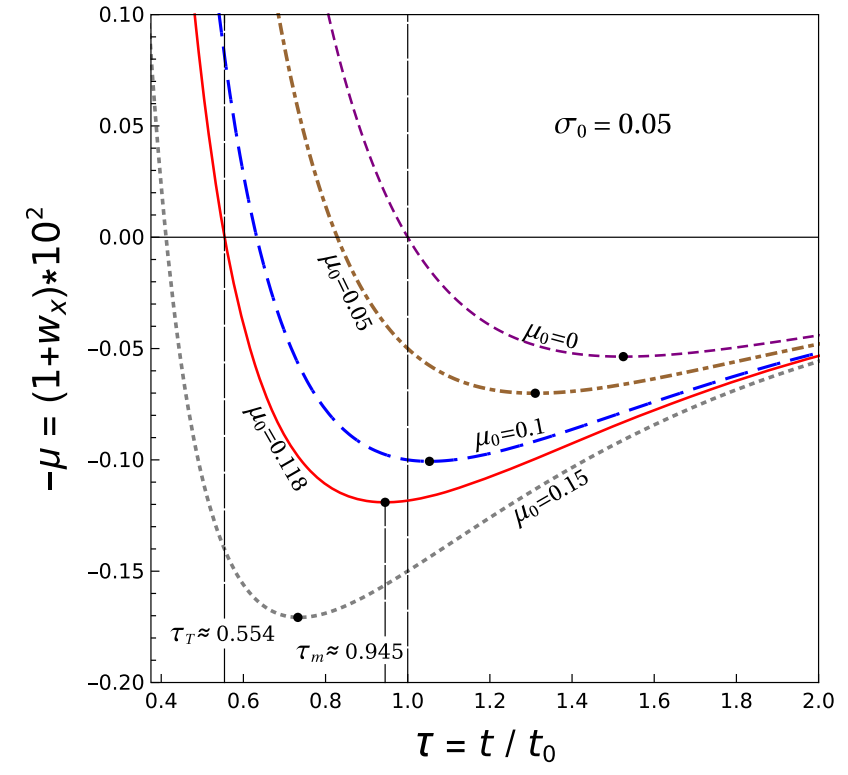

(a) $-\mu$ vs $t / t_{0}$ for $\sigma_{0}=0.05$ and $\mu_{0} \in(0,0.15)$.

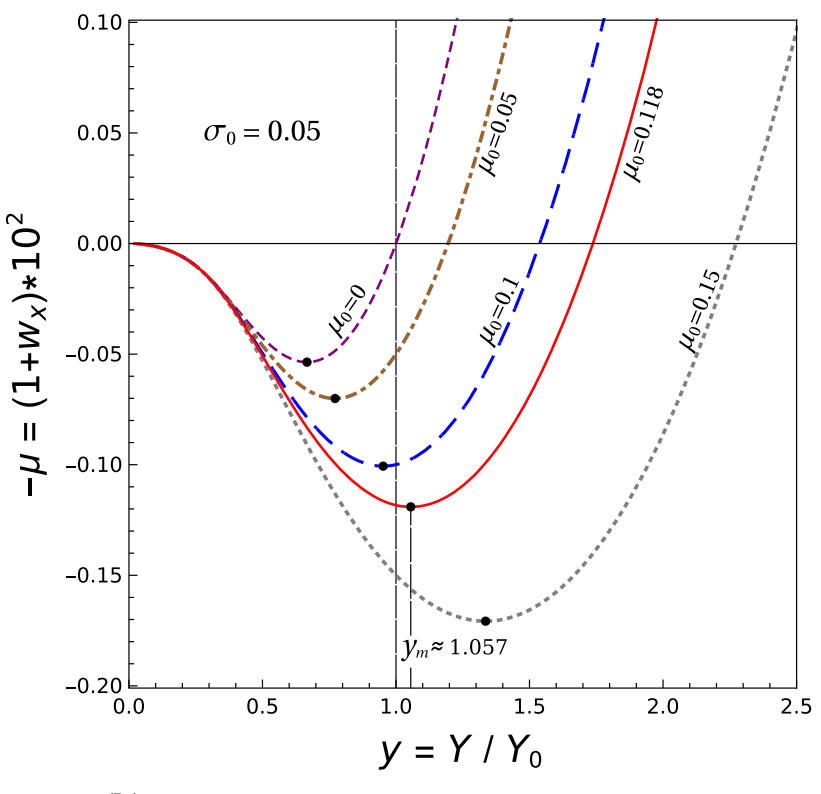

(b) $-\mu$ vs $Y / Y_{0}$ for $\sigma_{0}=0.05$ and $\mu_{0} \in(0,0.15)$.

Fig. 1 Variation of the percentage change in the effective DE equation of state: $-\mu=100\left(1+w_{X}\right)$, with a the rationalized cosmic time $\tau=t / t_{0}$, and $\mathbf{b}$ the rationalized correction $y=Y / Y_{0}$ in the MMT potential, for a fixed parametric value $\sigma_{0}=0.05$ and discrete values of the parameter $\mu_{0} \in(0,0.15)$. The thick dots indicate the minima of the plots

$$
\begin{aligned}
\frac{\tanh \left(\zeta_{0} \tau_{m}\right)}{2 \zeta_{0} \tau_{m}}= & -16 \tau_{m} \mathrm{w}_{X}^{(1)}\left(\tau_{m}\right) \\
\approx & \frac{\zeta_{0} \tau_{m}}{\sinh ^{2}\left(\zeta_{0} \tau_{m}\right)}\left[\left(1+\frac{4 \mu_{0}}{25 \sigma_{0}}\right) \frac{\sinh ^{2} \zeta_{0}}{\zeta_{0}}\right. \\
& \left.+\operatorname{Shi}\left(2 \zeta_{0} \tau_{m}\right)-\operatorname{Shi}\left(2 \zeta_{0}\right)\right]-1
\end{aligned}
$$

or, equivalently, by virtue of the relationship(4.27), 


$$
\begin{aligned}
& {\left[1+\frac{\tanh \left(\zeta_{0} \tau_{m}\right)}{2 \zeta_{0} \tau_{m}}\right] \frac{\sinh ^{2}\left(\zeta_{0} \tau_{m}\right)}{\zeta_{0} \tau_{m}}-\operatorname{Shi}\left(2 \zeta_{0} \tau_{m}\right)} \\
& \approx \frac{\sinh ^{2}\left(\zeta_{0} \tau_{C}\right)}{\zeta_{0} \tau_{C}}-\operatorname{Shi}\left(2 \zeta_{0} \tau_{C}\right) .
\end{aligned}
$$

Solving this equation numerically, for $\zeta_{0}=1.1881$, we find $\tau_{m}=0.945$ in the optimal case $\tau_{C}=\tau_{T}=0.554$ (or equivalently $\mu_{0}=2.367 \sigma_{0}$ ), without any allusion to the typical fixation of $\mu_{0}$ or $\sigma_{0}$.

It is also worth examining how the percentage correction $-\mu$ varies with the quantity $y(t) \equiv Y(t) / Y_{0}$, where $Y_{0} \equiv Y\left(t_{0}\right)$. After all, it is the specific form of the fractional correction in the potential, $Y(t)$, given by Eq. (4.4), which makes the cosmic super-acceleration plausible. Now, as we see from Fig. 1b, the criterion $t_{C} \in\left(t_{T}, t_{0}\right)$, and correspondingly the viable range of $\mu_{0}$ (for a fixed $\sigma_{0}$ ), could be ascribed to the valid zone of occurence of $y=y_{m}$, i.e. the point at which $-\mu$ is minimized. In particular, the above bound $\mu_{0} \lesssim 2.367 \sigma_{0}$ holds only for $y_{m} \lesssim 1.057$, thus implying that the $-\mu(y)$ variation should be such that the minimum point $y_{m}$ is reached no earlier than an epoch just surpassing the present epoch. This is nonetheless a general criterion for the viability of the phantom crossing (not specific to the choice $\sigma_{0}=0.05$ ). Figure $1 \mathrm{~b}$ also illustrates the nontransience of the phantom regime, i.e. the super-acceleration is ever-lasting (although it slows down progressively after reaching $y_{m}$ ). Analytically, this follows from Eq. (4.4) which implies $y \equiv Y / Y_{0} \approx \tau^{-1}$. Therefore, $y$ tends to vanish in the asymptotic limit $(\tau \rightarrow \infty)$, and so does the correction $\mu$.

II. If, on the other hand, we resort to a fixed value of $\mu_{0}$, say a fairly low one $(=0.05)$, then as illustrated in Fig. $2 \mathrm{a}$, and as derived from Eq. (4.27), a realistic phantom barrier crossing requires a lower limit on the parameter $\sigma_{0}$, close to (but greater than) 0.02 , for the $\zeta_{0}$ and $\tau_{T}$ estimated above.

However, if we aspire to get a much larger $\mu_{0}$, say $\sim 0.5$ (i.e. about $\frac{1}{2} \%$ correction in $\mathrm{w}_{X}$ ), then by Eq. (4.27), we require $\sigma_{0} \gtrsim 0.211$, which may nonetheless compel us to go beyond the linear approximation. The above arguments concerning the criterion $t_{C} \in\left(t_{T}, t_{0}\right)$ being ascribed to the domain of appearance of the turning points $\tau=\tau_{m}$ would apply here as well. Also for completeness, it is worth examining the variation of $-\mu$ with $y=Y / Y_{0}$, for $\mu_{0}$ fixed at 0.05 and various parametric values of $\sigma_{0}$. The corresponding plots shown in Fig. $2 \mathrm{~b}$ display the same non-transient phantom regime as illustrated in Fig. $1 \mathrm{~b}$ above.

\section{Evolving torsion parameters and the extent of the Super-acceleration}

Let us recall the expressions (2.17) and (2.18) for the relevant torsion parameters, viz. the norms of the torsion mode vectors

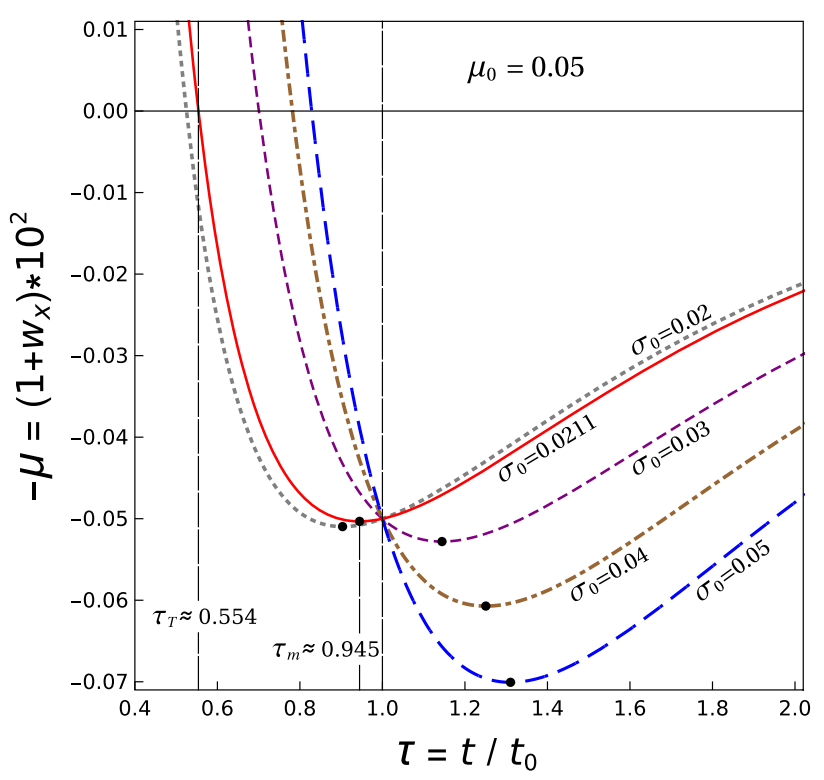

(a) $-\mu(t)$ vs $t / t_{0}$ for $\mu_{0}=0.05$ and $\sigma_{0} \in(0.02,0.05)$.

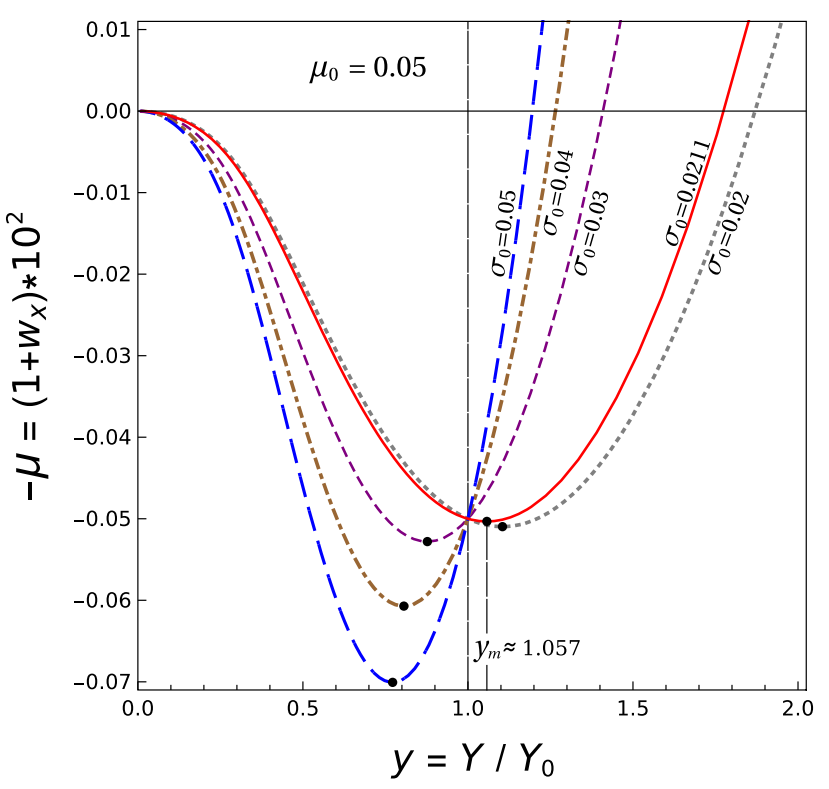

(b) $-\mu(t)$ vs $Y / Y_{0}$ for $\mu_{0}=0.05$ and $\sigma_{0} \in(0.02,0.05)$.

Fig. 2 Variation of the percentage change in the effective DE equation of state: $-\mu=100\left(1+\mathrm{w}_{X}\right)$, with a the rationalized cosmic time $\tau=t / t_{0}$, and $\mathbf{b}$ the rationalized correction $y=Y / Y_{0}$ in the MMT potential, for a fixed parametric value $\mu_{0}=0.05$ and discrete values of the parameter $\sigma_{0} \in(0.02,0.05)$. The thick dots indicate the minima of the plots

$\mathcal{T}_{\mu}$ and $\mathcal{A}_{\mu}$. With the MMT coupling function $\beta(\phi)$ given by Eq. (2.19), and the identification $s \gamma^{2}=\phi \equiv t$, such expressions reduce to

$$
\begin{aligned}
& \mathcal{T}^{2} \equiv-g^{\mu \nu} \mathcal{T}_{\mu} \mathcal{T}_{\nu}=\frac{9}{t^{2}}\left[1-\frac{s}{4(t+s))}\right]^{2}, \\
& \mathcal{A}^{2} \equiv-g^{\mu \nu} \mathcal{A}_{\mu} \mathcal{A}_{v}=\frac{9 s}{t(t+s)^{2}} .
\end{aligned}
$$


Our interest, of course, is in getting a quantitative measure of the extent to which the scenario in Paper 1 is modified when the parameter $s \neq 0$. For this we require a careful examination of the time-evolution of

$U_{T}:=\frac{\mathcal{T}^{2}-\overline{\mathcal{T}}^{2}}{\overline{\mathcal{T}}^{2}}$ and $U_{A}:=\frac{\mathcal{A}^{2}}{\overline{\mathcal{T}}^{2}}$

which are the fractional changes over the only existent torsion parameter in Paper 1, viz.

$\overline{\mathcal{T}}^{2} \equiv\left[-g^{\mu \nu} \mathcal{T}_{\mu} \mathcal{T}_{\nu}\right]_{s=0}=\frac{9}{t^{2}}$

that had led to the $\Lambda \mathrm{CDM}$ solution therein [191].

Using Eqs. (5.1), (5.2) and (5.4), along with the definitions (4.2), we can write

$U_{T}(\tau)=-\frac{U_{A}(\tau)}{2}\left(1+\frac{7 \sigma_{0}}{8 \tau}\right)$,

$U_{A}(\tau)=\frac{\sigma_{0} \tau}{\left(\tau+\sigma_{0}\right)^{2}}$,

and consequently re-express the fractional correction to the effective potential $\bar{W}=2 \Lambda$ of Paper 1 as

$Y(\tau)=\frac{\sigma_{0}}{16\left(\tau+\sigma_{0}\right)}=-\frac{U_{A}(\tau)+16 U_{T}(\tau)}{112}$.

Note that so far there is no approximation - all the relations (5.5)-(5.7) are exact. Nevertheless, from the perspective of our analysis in the previous section (anticipating per se, mild deviations from $\Lambda \mathrm{CDM}$ due to the MMT extension), we shall only consider small values of the parameter $\sigma_{0}$ from here on.

Now for small $\sigma_{0}$, the fractional corrections $U_{A}$ and $U_{T}$, although decrease with time, remain of the same order of magnitude as $\sigma_{0}$ itself, in the temporal range of interest $t \in$ $\left(t_{T}, t_{0}\right)$, or $\tau \in\left(\tau_{T}, 1\right)$. However, the resulting fractional change in the potential, $Y$, turns out be comparatively much smaller, as illustrated with two fiducial settings $\sigma_{0}=0.05$ and $\sigma_{0}=0.1$ in Fig. 3a, b respectively. This smallness of $Y$, and as a consequence the weak time-variation of the effective DE equation of state parameter $\mathrm{w}_{X}$, of course justifies the linear approximation in the preceding section. Note also that the peak values of $U_{A},\left|U_{T}\right|$ and $Y$ are independent of the value of $\sigma_{0}$. In particular, the above expression for $U_{A}$ implies that the latter attains its maximum value $(=0.25)$ at $\tau=\sigma_{0}$, as illustrated in Fig. 3a, b. On the other hand, $\left|U_{T}\right|$ and $Y$ being monotonically decreasing functions of $\tau$, have their maximum values (equal to 0.4375 and 0.0625 respectively) at $\tau=0$.

As to the values of $U_{A},\left|U_{T}\right|$ and $Y$ at the present epoch $t=t_{0}$, it is evident from Eqs. (5.5)-(5.7) that all of them are

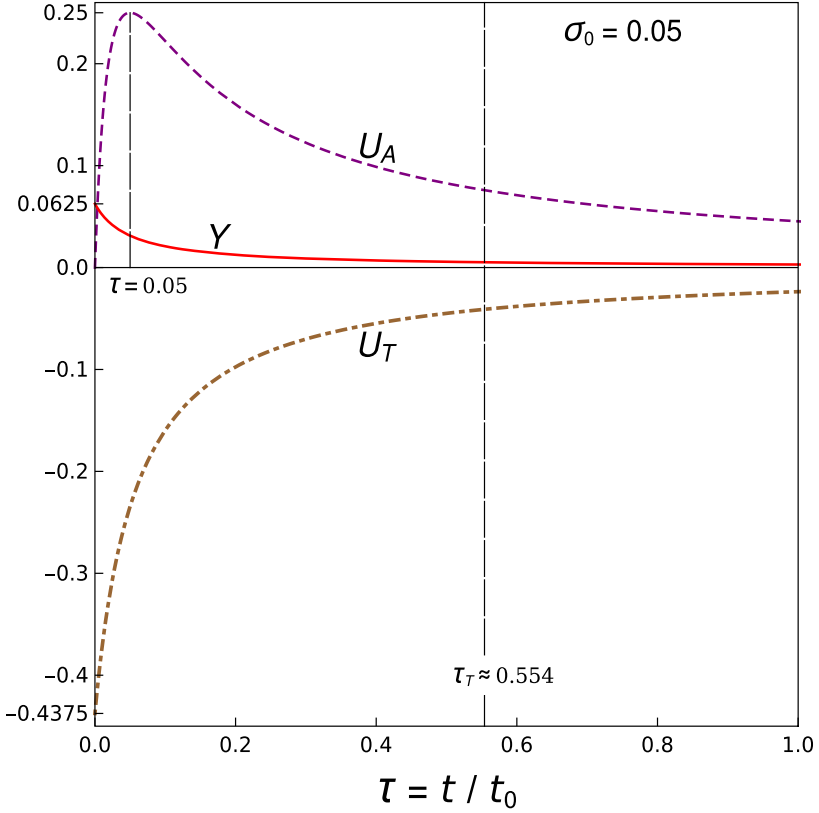

(a) $U_{A}, U_{T}$ and $Y$ vs $t / t_{0}$ for $\sigma_{0}=0.05$.

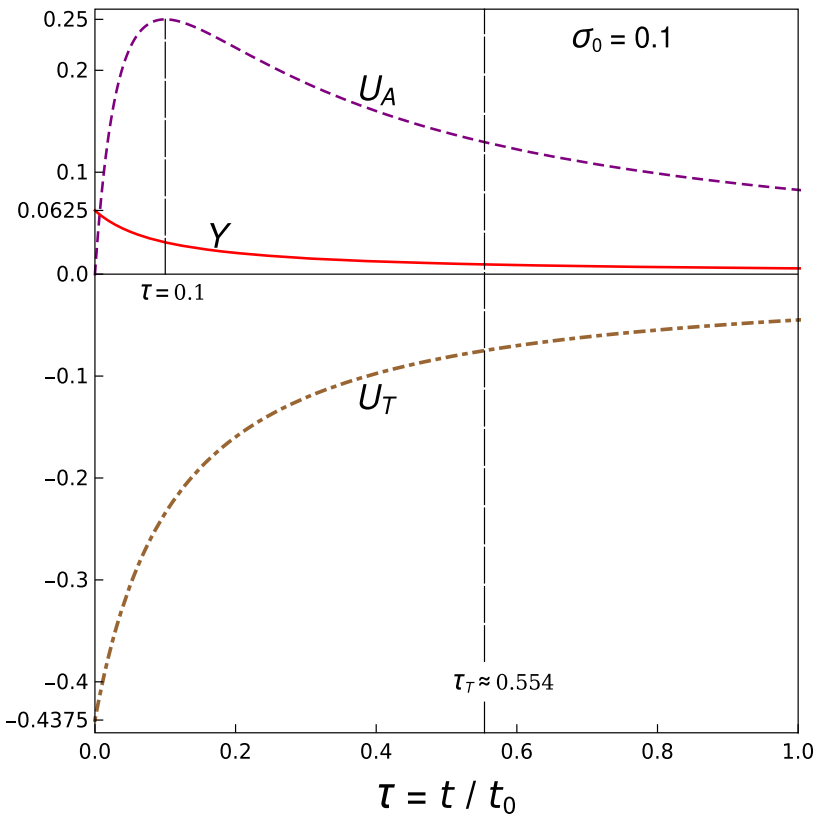

(b) $U_{A}, U_{T}$ and $Y$ vs $t / t_{0}$ for $\sigma_{0}=0.1$.

Fig. 3 Variations of the fractional changes in the torsion parameters, viz. $U_{A}(\tau)$ and $U_{T}(\tau)$, and their resultant $Y(\tau)$, for fixed parametric values: $\mathbf{a} \sigma_{0}=0.05$ and $\mathbf{b} \sigma_{0}=0.1$

smaller than $\sigma_{0}$, for $0<\sigma_{0} \lesssim 1$ :

$$
\begin{gathered}
U_{0}^{A} \equiv U_{A}\left(t_{0}\right)=\sigma_{0}\left[1+\varepsilon_{0}^{A}\right], \\
\left|U_{0}^{T}\right| \equiv\left|U_{T}\left(t_{0}\right)\right|=\frac{\sigma_{0}}{2}\left[1+\varepsilon_{0}^{T}\right], \\
Y_{0} \equiv Y\left(t_{0}\right)=\frac{\sigma_{0}}{16}\left[1+\varepsilon_{0}^{Y}\right],
\end{gathered}
$$




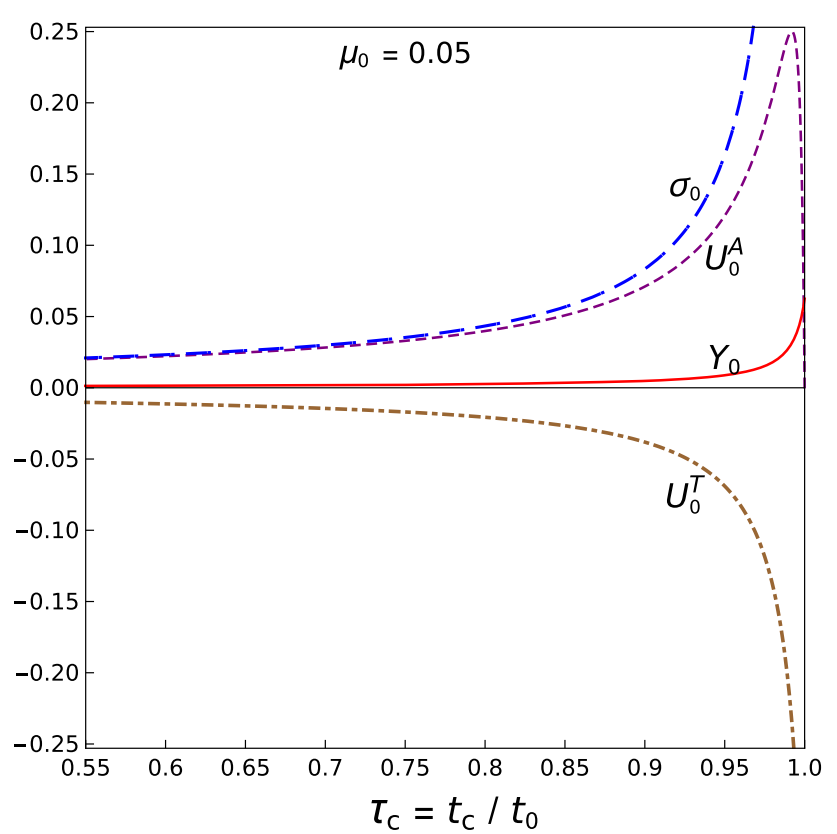

(a) $\sigma_{0}, U_{0}^{A}, U_{0}^{T}$ and $Y_{0}$ vs $t_{C} / t_{0}$ for $\mu_{0}=0.05$.

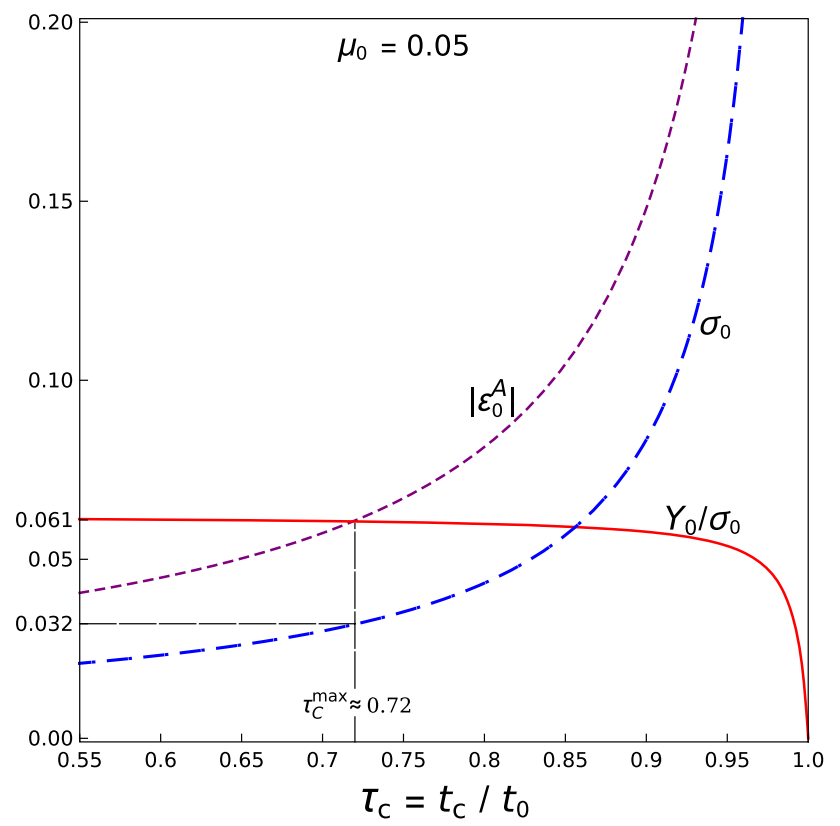

(b) $\sigma_{0}, Y_{0} / \sigma_{0}$ and $\left|\varepsilon_{0}^{A}\right|$ vs $t_{C} / t_{0}$ for $\mu_{0}=0.05$.

Fig. 4 Approximated variations of the parametric values of $\mathbf{a} \sigma_{0}$ and the fractional changes in the torsion parameters at the present epoch, viz. $U_{0}^{A}$ and $U_{0}^{T}$, as well as their resultant $Y_{0}$, and $\mathbf{b} \sigma_{0}, Y_{0} / \sigma_{0}$ and $\left|\varepsilon_{0}^{A}\right|$, i.e. the fractional deviation of $U_{0}^{A}$ from $\sigma_{0}$, with the rationalized phantom crossing time $\tau_{C}=t_{C} / t_{0}$ in the viable range $(0.55,1)$, for a fixed $\mu_{0}=0.05$

where

$$
\varepsilon_{0}^{A}=-\frac{\sigma_{0}\left(2+\sigma_{0}\right)}{\left(1+\sigma_{0}\right)^{2}}=-2 \sigma_{0}+\mathcal{O}\left(\sigma_{0}^{2}\right),
$$

$$
\begin{aligned}
& \varepsilon_{0}^{T}=-\frac{\sigma_{0}\left(9+8 \sigma_{0}\right)}{8\left(1+\sigma_{0}\right)^{2}}=-\frac{9 \sigma_{0}}{8}+\mathcal{O}\left(\sigma_{0}^{2}\right), \\
& \varepsilon_{0}^{Y}=-\frac{\sigma_{0}}{1+\sigma_{0}}=-\sigma_{0}+\mathcal{O}\left(\sigma_{0}^{2}\right)
\end{aligned}
$$

Now, using Eq. (4.27) one can determine how $\sigma_{0}$, and hence $U_{0}^{A},\left|U_{0}^{T}\right|$ and $Y_{0}$, vary approximately with the rationalized phantom crossing time $\tau_{C}\left(={ }^{t} / t_{0}\right)$, for a fixed value of the percentage correction $\mu_{0}$ in $\mathrm{w}_{X}$ at $t=t_{0}$ (or $\tau=1$ ). Figure $4 \mathrm{a}$ shows such parametric variations, within the viable range $t_{C} \in\left(t_{T}, t_{0}\right)$, or $\tau_{C} \in(0.55,1)$, for a fiducial setting $\mu_{0}=$ 0.05 (which nonetheless is well within the $1 \sigma$ error estimates from recent observations [230]).

A stringent upper limit can in principle be placed on $t_{C}$, and hence on $\sigma_{0}$, for a given value of $\mu_{0}$, from the argument that $Y_{0}$ would be of significance only when it is $\gtrsim \sigma_{0}\left|\varepsilon_{0}^{A}\right|$, where $\left|\varepsilon_{0}^{A}\right|$ is the absolute fractional deviation of $U_{0}^{A}$ from $\sigma_{0}$. Specifically, this could be seen as a consequence of a stringent limit being imposed on the extent of the linear approximation, for which $U_{0}^{A} \approx \sigma_{0}$, by Eq. (5.8). The higher order change, i.e. the deviation of the exact $U_{0}^{A}$ from $\sigma_{0}$, given by the amount $\sigma_{0}\left|\varepsilon_{0}^{A}\right|$, need to be $\lesssim Y_{0}$, because otherwise $Y_{0}$ would also be a higher order effect, which would in turn imply the invalidity of the linear approximation ${ }^{13}$. As shown in Fig. 4b, the effect of $Y_{0}$ of a very small amount $\left(\lesssim 0.061 \sigma_{0}\right)$, could yet be perceptible in the linear approximation (i.e. satisfy the condition $\left.Y_{0} \gtrsim \sigma_{0}\left|\varepsilon_{0}^{A}\right|\right)$ and lead to a reasonably significant amount of $\mu_{0}(=0.05)$, only if $t_{C} \lesssim 0.72 t_{0}$, or $\sigma_{0} \lesssim 0.032$ correspondingly. In principle, a lesser upper bound on $\sigma_{0}$, culminating to an even smaller amount of $Y_{0}$, may provide a more significant $\mu_{0}$, however at the expense of reducing the upper limit of $t_{C}$. One can therefore infer the following:

- As the phantom crossing time $t_{C}$ cannot be smaller than the deceleration-to-acceleration transition time $t_{T}$, the percentage correction $\mu_{0}$ (in the effective DE equation of state $\left.\mathrm{w}_{X}\left(t_{0}\right)\right)$ cannot get enhanced much further beyond the value 0.05 , at least in the linear approximation.

- Nevertheless, the extended MMT-cosmological scenario seems more attractive for a $t_{C}$ value closer to $t_{T}$, i.e. for an earlier commencement of the super-accelerating regime, from the point of view of having larger value of $\mu_{0}$ with lesser strength of coupling $\sigma_{0}$ of the mimetic field with the Holst term. In other words, the weaker the MMT extension, the stronger is the effect in $\mathrm{w}_{X}\left(t_{0}\right)$. There is a limit to this of course, since $t_{C}$ must not fall short of $t_{T}$.

\footnotetext{
13 One can alternatively consider imposing $Y_{0} \gtrsim \sigma_{0}\left|\varepsilon_{0}^{T}\right|$ or $Y_{0} \gtrsim \sigma_{0}\left|\varepsilon_{0}^{Y}\right|$, however the resulting upper bound on $\sigma_{0}$ (or $t_{C}$ ) would then be less stringent, since both $\left|\varepsilon_{0}^{T}\right|$ and $\left|\varepsilon_{0}^{Y}\right|$ are smaller than $\left|\varepsilon_{0}^{A}\right|$, by Eqs. (5.11)(5.13).
} 


\section{Conclusion}

A viable phantom crossing evolution of a unified cosmological dark sector is thus demonstrated by extending the basic mimetic-metric-torsion (MMT) formalism with an explicit coupling of the mimetic field $\phi$ and the Holst term, motivated from the following:

Firstly, its compliance with the basic precept of MMT gravity, viz. the preservation of conformal symmetry while letting $\phi$ to manifest geometrically as the source of torsion (or certain mode(s) thereof).

Secondly, and most crucially, its instrumentality in making torsion's main characteristic, viz. the anti-symmetry, accountable for the dynamical evolution of a given physical system.

To be more specific, the reason why we have incorporated the $\phi$-coupled Holst term is that, apart from keeping the conformal symmetry of the MMT theory unaffected, it ideally meets our objective of perceiving a plausible effect of the axial (or pseudo-trace) mode $\mathcal{A}_{\mu}$ of torsion, in addition to that of the latter's trace mode $\mathcal{T}_{\mu}$, on the cosmic evolution profile. In particular, for clarity in interpreting results, irrespective of the explicit $\phi$-coupling, we have preferred to resort to a non-topological characterization of the bare Holst term by promoting its coefficient, viz. the Barbero-Immirzi (BI) parameter $\gamma$, to the status of a field. Considering this field to be a (presumably primordial) pseudo-scalar (or, an axion), we could avoid gravitational parity violation, and hence a direct confrontation with the latter's miniscule observational signature at cosmological scales. Consequently, an identification of $\phi$ with $\gamma^{2}$ (i.e. the simplest possible even function of $\gamma$ ), using a scalar Lagrange multiplier field, had made it evident that both the (a priori independent) torsion modes $\mathcal{T}_{\mu}$ and $\mathcal{A}_{\mu}$ are induced by $\phi$. In the paradigm of the standard FRW cosmology, the outcome is in some sense, a 'geometric unification' of the dark sector, as both the effective DM and $\mathrm{DE}$ components of the universe, being purportedly the artefacts of $\mathcal{T}_{\mu}$ and $\mathcal{A}_{\mu}$, have had their dynamics driven by the same scalar field $\phi$.

Technically of course, the MMT theory (introduced in Paper 1) is designed to have almost all of its weight resting on the pre-assigned contact coupling, $\beta(\phi)$, between the mimetic field and the entire torsion-dependent part of the Riemann-Cartan $\left(U_{4}\right)$ Lagrangian. In the extended formulation, such a coupling obviously carries to the Holst term, which augments the $U_{4}$ Lagrangian. The further postulation of a pseudo-scalar BI field, and subsequently the identification $\phi=s \gamma^{2}$ (where $s$ is a dimensionless constant), are only expected to lead to a subtle modification of what $\beta(\phi)$ alone can inflict on a given system configuration. Nevertheless, there arises the important question as to whether such a modification, despite its suppressed quantitativeness, can turn out to be significant in the cosmological context. This is what we have addressed specifically via our analysis in the preceding sections.

In particular, taking the MMT coupling function in the form $\beta(\phi) \sim \phi^{2}$, we have brought the time-variance of the effective mimetic potential $W(\phi)$ in the reckoning, as opposed to its constancy in Paper 1 , viz. $W=\bar{W}=2 \Lambda$, due to the only existent torsion mode $\mathcal{T}_{\mu}$ (in absence of the Holst term) therein. While the coupling $\beta(\phi) \sim \phi^{2}$ has had its motivation from the point of view of its natural appearance in metric-torsion theories involving scalar field(s) [97, 148150], the effect of the typical functional form it ascribes to the fractional modification $Y(\phi)$ of the constant potential $\bar{W}=2 \Lambda$ (of Paper 1) has turned out to be quite fascinating in the cosmological context. Specifically, such a $\phi^{2}$-coupling implies an inverse functional dependence of $Y$ on $\phi$, or equivalently, on the cosmic time $t$ in the FRW framework. The consequence of this, by virtue of the mimetic constraint, is a super-accelerating phase of cosmic evolution, marked by the diminution of the effective DE equation of state parameter $\mathrm{w}_{X}(t)$ below the $\Lambda \mathrm{CDM}$ value -1 . In fact, as we have inferred via a set of arguments, the super-acceleration is always plausible in our extended MMT scenario, which most importantly, does not appear to have any theoretical obstacles, such as those concerning ghost or phantom degrees of freedom. This may be understood from the point of view that none of the energy conditions, except the strong one, is violated in our entire formalism. The violation of the strong energy condition is of course nothing unusual - it is mandated for any attempt of explaining the late-time cosmic acceleration (regardless of an even later super-acceleration) in the standard FRW framework.

Nevertheless, despite having the theoretical consistency, there remained the task for us to assert whether the transition from $\mathrm{w}_{X}>-1$ to $\mathrm{w}_{X}<-1$, or the phantom barrier crossing, is at all physically realizable, and if so, under what condition(s). In specific terms, a physically realistic (or viable) phantom crossing implies that the epoch of its occurence, $t=t_{C}$, has to be within the time-span $\left(t_{T}, t_{0}\right)$, where $t_{T}$ denotes the deceleration-to-acceleration transition epoch and $t_{0}$ denotes the present epoch. While the lower bound $t_{C}>t_{T}$ is obvious, the upper bound $t_{C}<t_{0}$ actually pertains to a fair amount of observational signification of a mild superacceleration to have commenced in the near past phase of evolution of the universe, and continuing to the present epoch and beyond [230]. The mildness of course alludes to the gross observational concordance on $\Lambda \mathrm{CDM}$ cosmology. That is to say, in whichever way the DE equation of state parameter $\mathrm{w}_{X}$ may deviate from the $\Lambda \mathrm{CDM}$ value -1 , observations disfavour the latter's error limits to be breached to any perceivable extent. Now, to get the condition(s) for $t_{C} \in\left(t_{T}, t_{0}\right)$ to hold (if at all), it is imperative to determine the functional form of $\mathrm{w}_{X}(t)$ by explicitly solving the corresponding cosmological equations. However, instead of attempting an exact 
analytic solution, we have adopted an approximation method, upon treating the effect of the function $Y(\phi)$ as a small perturbation over that due to the constant potential $\bar{W}=2 \Lambda$, viz. the $\Lambda \mathrm{CDM}$ solution in Paper 1 . Admittedly, the strong observational support for $\Lambda \mathrm{CDM}$ has made it reasonable for us to assume that $Y(\phi)$ would always inflict a small deviation of $\mathrm{w}_{X}$ from the value -1 .

Working out $\mathrm{w}_{X}(t)$ in the approximated form, we have shown that the phantom crossing is indeed physically realizable, however for a severely restricted range of values of our MMT model parameters, viz. $\sigma_{0}$ and $\mu_{0}$, where $\sigma_{0}=s / t_{0}$ and $\mu_{0}$ is the fractional amount by which $\mathrm{w}_{X}$ differs from -1 at $t=t_{0}$. In particular, limiting our analysis to the linear order of the (presumably small) parameter $\sigma_{0}$, we have illustrated the time-evolution of $\mathrm{w}_{X}$ for certain fiducial settings of either a fixed $\sigma_{0}$ or a fixed $\mu_{0}$. Strikingly enough, all such settings have made the revelation of the phantom regime being non-transient, i.e. the super-acceleration is ever-lasting, even though it slows down progressively after reaching an optimum point. A close inspection of the evolution profiles of the torsion parameters, viz. the norms of the torsion trace and pseudo-trace mode vectors $\mathcal{T}_{\mu}$ and $\mathcal{A}_{\mu}$, have enabled us to determine the validity of the linear approximation, and hence the extremity of the phantom crossing time $t_{C}$, as well as that of $\mathrm{w}_{X}\left(t_{0}\right)$ (or equivalently, $\mu_{0}$ ). In fact, the latter is found to be not exceeding a value typically well within the Planck 2018 TT,TE,EE+lowE+Lensing+BAO error estimates for $\Lambda \mathrm{CDM}$ [230]. This nonetheless signifies a very low extent of the super-acceleration, in well accord with our presumption of its smallness.

On the whole, we have seen some really appealing outcomes of our entire program of extending the basic MMT formalism of Paper 1. Not only that it consolidates the tantalizing picture of a geometrically unified dark sector we have had therein, but also discerns the viability of the cosmic super-acceleration, or the phantom phase, without any impending danger of dealing with a ghost-like entity. This may nonetheless be counted towards the robustness of the emergent cosmological model, since after all, from a purely theoretical standpoint, a provision for the phantom barrier crossing is desirable on account of the flexibility thus comprehended while making statistical estimations of the model parameters using the observational data.

Nevertheless, apart from the technical difficulties the extreme mildness of the cosmic super-acceleration may pose in its detection with the current generation of observational probes, there remain several issues to be pondered on. The foremost, of course, are those concerning the Ostrogradsky ghost or(and) gradient instabilities at the perturbative level, due to the presence of higher derivative $\left(\sim(\square \phi)^{2}\right)$ extension of the MMT Lagrangian [25-27]. A plausible removal of such instabilities is demonstrated, via a proposed alternative extension, in the Appendix below. Secondly, there is the associated problem of the caustic singularities, which in fact affects not only the mimetic theory, but also any scalar-tensor model exhibiting the mimicry of a dust-like fluid description. Caustic formation is unavoidable for any constrained scalar field, such as the mimetic one, that leads to a dust velocity flow tangential to the time-like geodesics [58,193195]. Within the scope of mimetic gravity, this problem has been circumvented via vectorial (gauge field) extensions [5254]. However, in the MMT scenario the presence of the torsion coupling with the mimetic field $\phi$ may be of some aid, since it implies that apart from being a fundamental mode of gravity, the field $\phi$ manifests itself geometrically as the source of one (or more) vector mode(s) of torsion. Furthermore, the geodesics of actual importance here are the affine geodesics (or, the auto-parallel curves) which are in general not identical to the metric geodesics in the Riemann-Cartan $\left(U_{4}\right)$ space-time. Therefore, a careful inspection resulting in a chosen specific form of the $\phi$-coupling with the torsion vector mode $\mathcal{T}_{\mu}$ had enabled us to get an effective dustlike (MMT) fluid description in Paper 1 (see the Appendix therein). Admittedly however, a more rigorous contemplation of the focusing theorem and caustics is needed in this context, by taking note of the existent references [237,238], which is one of the assignments we have undertaken presently.

Other pertinent questions are, for instance, (i) can there be any obvious way of realizing the $\phi$-coupling with torsion, with or without involving the Holst term? (ii) would that be perceptible via the Hilbert-Palatini formulation of mimetic gravity? (iii) how would the magnitude of the superacceleration be affected if there be a constraint more complicated than (2.6)? (iv) can that constraint be imposed automatically (i.e. without the aid of a Lagrange multiplier) via some mechanism? Attempts of addressing to some of these are currently underway, and hopefully be reported soon.

Acknowledgements The authors acknowledge useful discussions with Mohit Sharma. The work of AD is supported by University Grants Commission (UGC), Government of India.

Data Availability Statement This manuscript has no associated data or the data will not be deposited. [Authors' comment: Only certain wellknown observational results is used in this paper. No actual analysis with raw data is carried out as such.]

Open Access This article is licensed under a Creative Commons Attribution 4.0 International License, which permits use, sharing, adaptation, distribution and reproduction in any medium or format, as long as you give appropriate credit to the original author(s) and the source, provide a link to the Creative Commons licence, and indicate if changes were made. The images or other third party material in this article are included in the article's Creative Commons licence, unless indicated otherwise in a credit line to the material. If material is not included in the article's Creative Commons licence and your intended use is not permitted by statutory regulation or exceeds the permitted use, you will need to obtain permission directly from the copyright holder. To view a copy of this licence, visit http://creativecomm 
ons.org/licenses/by/4.0/.

Funded by SCOAP ${ }^{3}$.

\section{Appendix: A note on the possible aversion of ghost and gradient instabilities}

Let us refer back to the action (2.2). Apart from the matter action $S^{(m)}$, it has the pure MMT action with the general structure

$S_{M M T}=\int d^{4} x \sqrt{-g}\left[\mathcal{L}^{(g)}+\mathcal{L}^{(c)}+\mathcal{L}^{(h)}\right]$,

that includes the purely gravitational part (alongwith the $\phi$ coupled torsional contributions) described by the Lagrangian

$\mathcal{L}^{(g)}=\frac{1}{2}\left[R+\beta(\phi)\left(\Theta+\frac{{ }^{\star} \Theta}{2 \gamma}\right)\right]$,

the constraint part with Lagrangian

$\mathcal{L}^{(c)}=\frac{1}{2}\left[\lambda \cdot(X-1)+v \cdot\left(\phi-s \gamma^{2}\right)\right]$,

and the higher derivative (HD) augmentation, described by the Lagrangian

$\mathcal{L}^{(h)}=\frac{\alpha}{2}(\square \phi)^{2}$.

As is well-known, such an augmentation crucially leads to a non-zero sound speed of the mimetic matter density perturbations, given by [2]

$c_{s}=\sqrt{\frac{\alpha}{2-3 \alpha}}$,

however, at the expense of making the mimetic field dynamical. While a non-zero $c_{s}$ is essential for defining the usual quantum fluctuations due to $\phi$, so that the latter can provide the seeds of the observed large scale structure of the universe [2,3], the presence of a HD term in the action is in general problematic as it leads to Ostrogradsky instabilities. Remarkably, the mimetic constraint $X=1$ comes to the rescue here, as it eliminates the dynamical mode arising from $(\square \phi)^{2}$, and in turn the Ostrogradsky ghost, for a suitable range of values of the parameter $\alpha$ [2]. However, a rather critical inspection reveals that there remains a stringent problem of a gradient instability, which requires to be confronted with substantial rigour [25-27]. As demonstrated by several authors in recent times, such a problem could be remedied by introducing an explicit contact coupling of an appropriate function of $\square \phi$ (and possible other HD constructs, e.g. $\phi_{\mu \nu} \phi^{\mu \nu}$ where $\left.\phi_{\mu \nu} \equiv \nabla_{\mu} \nabla_{\nu} \phi\right)$ with curvature invariants, such as $R$ [26-29]. Nevertheless, this still implies a dynamical $\phi-$ the assumption which we endeavor to disregard in what follows.

To be specific, we consider a scenario in which the pure MMT action (6.1) has the same gravitational and constraint Lagrangians given respectively by (6.2) and (6.3), but the HD Lagrangian (6.4) replaced by

$\mathcal{L}^{(h)}=\frac{1}{2}\left[\phi \square \psi-m^{2} \psi^{2}+n \psi R-\ell^{2} R^{2}\right]$,

where $\psi$ is a dynamical scalar field (considered dimensionless), and $n, \ell, m$ are constants ( $n$ is dimensionless, whereas $\ell$ and $m$ have unit length and mass dimensions, respectively). Eq. (6.6) essentially means that we are considering a theory with two scalar fields - one is the non-dynamical mimetic field $\phi$ whereas the other is a (presumably primordial) dynamical field with a non-canonical kinetic term and having explicit contact couplings with both $\phi$ and $R$. The purpose of incorporating the $R^{2}$ term is to eliminate any scale dependence in the theory, as we shall see below. Note also that although the proposed Lagrangian (6.6) seems to have nothing to do with the presence of torsion, a correspondence could be traced, albeit with certain additional subtleties which are being examined in our subsequent works (in preparation) $[229,241]$. For the time being, however, in this paper, we consider the Lagrangian (6.6) to be just an exemplary one, that replaces Eq. (6.4) in an attempt of providing a nonzero $c_{s}$, and side-by-side removing the gradient instability, without requiring the mimetic field to be dynamical, to begin with.

Eliminating a surface term one can make $\psi$ an auxiliary field, whence the corresponding variation of the full action would lead to the constraint

$\psi=\frac{\square \phi+n R}{2 m^{2}}$.

Hence, implementing this and the other constraint (2.6), and proceeding in the same way as in Sect. 2, we get

$$
\begin{aligned}
S_{M M T}= & \frac{1}{2} \int d^{4} x \sqrt{-g}\left[(1+\epsilon \square \phi) R+\alpha(\square \phi)^{2}\right. \\
& +\lambda(X-1)-W(\phi) X],
\end{aligned}
$$

where $W(\phi)$ is as given by Eq. (2.21), and we denote

$\alpha \equiv \frac{1}{4 m^{2}}$ and $\epsilon \equiv \frac{\ell}{2 m}$,

upon choosing $n=2 m \ell$, so that the $R^{2}$ contribution gets cancelled (thereby removing the scale-dependence).

Let us now resort to the standard Arnowitt-Deser-Misner (ADM) decomposition of the line element for the perturbed 
FRW space-time

$d s^{2}=-N^{2} d t^{2}+h_{i j}\left(d x^{i}+N^{i} d t\right)\left(d x^{j}+N^{j} d t\right)$,

where $h_{i j}$ is the induced metric, and $N$ and $N^{i}$ are respectively the lapse function and the shift vector. It is convenient to choose the unitary gauge in which the scalar (mimetic) field perturbations $\delta \phi=0$, and the induced metric assumes the form

$h_{i j}(\boldsymbol{x}, t)=a^{2}(t) \exp \left[2 I_{i j} \zeta(\boldsymbol{x}, t)+\gamma_{i j}(\boldsymbol{x}, t)\right]$,

where $\zeta$ and $\gamma_{i j}$ respectively denote the scalar (or curvature) mode and the elements of the (transverse and trace-free) tensor mode of perturbations. As $\phi$ is purely a function of time in this gauge, the mimetic constraint $X=1$ implies

$N=\dot{\phi}, \quad \partial_{i} N=0$

Setting therefore, without loss of generality, $N=1$, as in ref. [27], we get

$\square \phi=-K$,

where $K=h^{i j} K_{i j}$ denotes the trace of the extrinsic curvature $K_{i j}=\frac{1}{2}\left[\dot{h}_{i j}-\left(\nabla_{i} N_{j}+\nabla_{j} N_{i}\right)\right]$.

The perturbed MMT action can hence be written, after certain simplifications, as

$$
\begin{aligned}
S_{M M T}= & \frac{1}{2} \int d^{4} x \sqrt{h}\left[(1-\epsilon K)\left\{{ }^{(3)} R+K_{i j} K^{i j}\right\}\right. \\
& \left.-(1-\alpha) K^{2}-W(\phi)\right]
\end{aligned}
$$

where $h$ is the determinant of the induced metric $h_{i j}$ and ${ }^{(3)} R$ denotes the corresponding curvature scalar. Varying this action with respect to the shift vector $N^{i}$ we get the corresponding equation of motion, given up to the first order in the perturbations, as

$\partial_{i} N^{i}=\frac{2 \epsilon \partial^{2} \zeta+[2(1-\epsilon K)-3(\alpha-5 \epsilon H)] a^{2}}{(\alpha-5 \epsilon H) a^{2}}$

which shows that $N^{i}$ only has a longitudinal part [27]. Following then the steps of ref. [27], and the general methodology of ref. [242], we obtain the quadratic order action (after a great deal of simplifications) as ${ }^{14}$

$$
\begin{aligned}
S_{M M T}^{(2)}= & \int d^{4} x a^{3}\left[f^{(T)}\left\{\dot{\gamma}_{i j} \dot{\gamma}^{i j}-e^{(T)} \partial_{i} \gamma_{j k} \partial^{i} \gamma^{j k}\right\}\right. \\
& +f^{(S)}\left\{\dot{\zeta}^{2}-e^{(S)} \partial_{i} \zeta \partial^{i} \zeta\right\} \\
& \left.+\tilde{f}^{(S)}\left(\partial^{2} \zeta\right)^{2}-\frac{1}{2} W(\phi)\right],
\end{aligned}
$$

where

$$
\begin{aligned}
f^{(T)}= & \frac{1-3 \epsilon H}{8}, \\
e^{(T)}= & a^{-2}, \\
f^{(S)}= & \frac{(1-3 \epsilon H)[3(\alpha-3 \epsilon H)-2]}{\alpha-5 \epsilon H}, \\
e^{(S)}= & -[3(\alpha-3 \epsilon H)-2]^{-1}[(\alpha-3 \epsilon H) \\
& \left.-2 \epsilon^{2}\left(\frac{3}{1-3 \epsilon H}-\frac{5}{\alpha-5 \epsilon H}\right) \dot{H}\right] a^{-2}, \\
\tilde{f}^{(S)}= & -\frac{2 \epsilon^{2} a^{-4}}{\alpha-5 \epsilon H},
\end{aligned}
$$

are the purely time-dependent coefficients of the tensor (T) and scalar (S) mode kinetic terms.

Ghost-freeness of the theory requires $f^{(T)}, f^{(S)}$ and $\tilde{f}^{(S)}$ to be positive definite at all epochs, and the gradient instabilities would be removed if $e^{(T)}$ and $e^{(S)}$ remain positive definite as well. Moreover, from Eq. (6.5) we see that $0 \leq c_{s}^{2} \leq 1$ implies $0 \leq \alpha \leq \frac{1}{2}$. Therefore, severe restrictions are bound to get imposed on the Hubble parameter $H(t)$, after being compounded with the demand that $\dot{H}(t)<0(\forall t)$, so that by the Raychaudhuri-Friedmann equation $\rho+p>0$, i.e. the null and weak energy conditions hold (see the discussion in the last paragraph of Sect. 3). A close inspection of Eqs. (6.17)-(6.21) nonetheless reveal that all the above criteria are fulfilled for the following bounds on the dimensionless Hubble rate

$\frac{\alpha}{5 \epsilon H_{0}} \leq \frac{H}{H_{0}} \leq \frac{\alpha}{3 \epsilon H_{0}}$.

So, to have the range of values of $H / H_{0}$ enhanced for a given $\alpha \in\left[0, \frac{1}{2}\right]$ and $H_{0}$, so as to bring more flexibility in the model, we require the parameter $\epsilon \ll \alpha$, in units of $H_{0}$. The smallness of $\epsilon$ implies a very weak coupling of $\square \phi$ with $R$, for which of course the background MMT cosmological evolution would not be affected much. Hence, our analysis in Sects. 3, 4 and 5 would still be valid, by and large.

\footnotetext{
14 The detailed calculations could be found in the subsequent papers [229,241], which we hope to report soon.
} 


\section{References}

1. A.H. Chamseddine, V. Mukhanov, JHEP 1311, 135 (2013)

2. A.H. Chamseddine, V. Mukhanov, A. Vikman, JCAP 1406, 017 (2014)

3. L. Sebastiani, S. Vagnozzi, R. Myrzakulov, Adv. High Energy Phys. 2017, 3156915 (2017)

4. E.J. Copeland, M. Sami, S. Tsujikawa, Int. J. Mod. Phys. D 15, $1753(2006)$

5. L. Amendola, S. Tsujikawa, Dark Energy: Theory and Observations (Cambridge University Press, Cambridge, 2010)

6. G. Wolschin, Lectures on Cosmology: Accelerated Expansion of the Universe (Springer, Berlin, 2010)

7. S. Matarrese, M. Colpi, V. Gorini, U. Moschella, Dark Matter and Dark Energy: A Challenge for Modern Cosmology (Springer, The Netherlands, 2011)

8. K. Bamba, S. Capozziello, S. Nojiri, S.D. Odintsov, Astrophys. Space Sci. 342, 155 (2012)

9. T. Chiba, Phys. Lett. B 575, 1 (2003)

10. S. Nojiri, S.D. Odintsov, Phys. Lett. B 631, 1 (2005)

11. S. Nojiri, S.D. Odintsov, Phys. Rev. D 74, 086005 (2006)

12. S. Nojiri, S.D. Odintsov, Int. J. Geom. Methods Mod. Phys. 04, 115 (2007)

13. S. Fay, R. Tavakol, S. Tsujikawa, Phys. Rev. D 75, 063509 (2007)

14. T.P. Sotiriou, V. Faraoni, Rev. Mod. Phys. 82, 451 (2010)

15. A. De Felice, S. Tsujikawa, Living Rev. Relativ. 13, 3 (2010)

16. T. Clifton, P.G. Ferreira, A. Padilla, C. Skordis, Phys. Rep. 513, 1 (2012)

17. E. Papantonopoulos, Modifications of Einstein's Theory of Gravity at Large Distances. Lecture Notes in Physics (Springer, Switzerland, 2015)

18. S. Nojiri, S.D. Odintsov, V.K. Oikonomou, Phys. Rep. 692, 1 (2017)

19. E.A. Lim, I. Sawicki, A. Vikman, JCAP 1005, 012 (2010)

20. C. Gao, Y. Gong, X. Wang, X. Chen, Phys. Lett. B 702(2-3), 107 (2011)

21. S. Capozziello, J. Matsumoto, S. Nojiri, S.D. Odintsov, Phys. Lett. B 693(2), 198 (2010)

22. Y. Cai, Y.-S. Piao, Phys. Rev. D 96(12), 124028 (2017)

23. M.A. Gorji, S.A.H. Mansoori, H. Firouzjahi, JCAP 1801, 020 (2018)

24. M. Chaichian, J. Kluson, M. Oksanen, A. Tureanu, JHEP 1412, 102 (2014)

25. A. Ijjas, J. Ripley, P.J. Steinhardt, Phys. Lett. B 760, 132 (2016)

26. S. Hirano, S. Nishi, T. Kobayashi, JCAP 1707, 009 (2017)

27. Y. Zheng, L. Shen, Y. Mou, M. Li, JCAP 1708, 040 (2017)

28. K. Takahashi, T. Kobayashi, JCAP 1711, 038 (2017)

29. H. Firouzjahi, M.A. Gorji, S.A.H. Mansoori, JCAP 1707, 031 (2017)

30. J. Ben Achour, D. Langlois, K. Noui, Phys. Rev. D 93, 124005 (2016)

31. D. Langlois, M. Mancarella, K. Noui, F. Vernizzi, JCAP 1902, 036 (2019)

32. R. Myrzakulov, L. Sebastiani, Astrophys. Space Sci. 361, 188 (2016)

33. D. Momeni, R. Myrzakulov, E. Gudekli, Int. J. Geom. Methods Mod. Phys. 12, 1550101 (2015)

34. A.V. Astashenok, S.D. Odintsov, V.K. Oikonomou, Class. Quantum Gravity 32, 185007 (2015)

35. E.H. Baffou, M.J.S. Houndjo, M. Hamani-Daouda, F.G. Alvarenga, Eur. Phys. J. C 77(10), 708 (2017)

36. F. Arroja, N. Bartolo, P. Karmakar, S. Matarrese, JCAP 1509, 051 (2015)

37. G. Cognola, R. Myrzakulov, L. Sebastiani, S. Vagnozzi, S. Zerbini, Class. Quantum Gravity 33, 225014 (2016)
38. M. Bouhmadi-Lopez, C.-Y. Chen, P. Chen, JCAP 1711, 053 (2017)

39. C.-Y. Chen, M. Bouhmadi-Lopez, P. Chen, Eur. Phys. J. C 78(1), 59 (2018)

40. N. Sadeghnezhad, K. Nozari, Phys. Lett. B 769, 134 (2017)

41. Y. Zhong, Y. Zhong, Y.-P. Zhang, Y.-X. Liu, Eur. Phys. J. C 78, 45 (2018)

42. Y. Zhong, Y.-P. Zhang, W.-D. Guo, Y.-X. Liu, JHEP 1904, 154 (2019)

43. A.H. Chemseddine, V. Mukhanov, JHEP 1806, 060 (2018)

44. A.H. Chemseddine, V. Mukhanov, JHEP 1806, 062 (2018)

45. O. Malaeb, C. Saghir, Eur. Phys. J. C 79(7), 584 (2019)

46. A.R. Solomon, V. Vardanyan, Y. Akrami, Phys. Lett. B 794, 135 (2019)

47. D. Momeni, A. Altaibayeva, R. Myrzakulov, Int. J. Geom. Methods Mod. Phys. 11, 1450091 (2014)

48. R. Myrzakulov, L. Sebastiani, S. Vagnozzi, S. Zerbini, Fundam. J. Mod. Phys. 8, 119 (2015)

49. N.A. Koshelev, Effective dark matter fluid with higher derivative corrections, e-print. arXiv:1512.07097

50. E.N. Saridakis, M. Tsoukalas, JCAP 1604, 017 (2016)

51. R. Kimura, A. Naruko, D. Yoshida, JCAP 1701, 002 (2017)

52. M.A. Gorji, S. Mukohyama, H. Firouzjahi, JCAP 1905, 019 (2019)

53. M.A. Gorji, S. Mukohyama, H. Firouzjahi, S.A. Hosseini Mansoori, JCAP 1808(8), 047 (2018)

54. P. Jiroušek, A. Vikman, JCAP 1904, 004 (2019)

55. A.H. Chamseddine, V. Mukhanov, T.B. Russ, Eur. Phys. J. C 79(7), 558 (2019)

56. A.H. Chamseddine, V. Mukhanov, T.B. Russ, Phys. Lett. B 798, 134939 (2019)

57. O. Malaeb, C. Saghir, Mimetic Horava Gravity and Surface terms, e-print. arXiv:2005.02469

58. A.O. Barvinsky, JCAP 1401, 014 (2014)

59. K. Hammer, A. Vikman, Many Faces of Mimetic Gravity, e-print arXiv: 1512.09118

60. M. Raza, K. Myrzakulov, D. Momeni, R. Myrzakulov, Int. J. Theor. Phys. 55, 2558 (2016)

61. J. Dutta, W. Khyllep, E.N. Saridakis, N. Tamanini, S. Vagnozzi, JCAP 1802, 041 (2018)

62. G. Leon, E.N. Saridakis, JCAP 1504, 031 (2015)

63. S.D. Odintsov, V.K. Oikonomou, Phys. Rev. D 93(2), 023517 (2016)

64. S. Nojiri, S.D. Odintsov, Mod. Phys. Lett. A 29, 1450211 (2014)

65. S. Nojiri, S.D. Odintsov, V.K. Oikonomou, Phys. Rev. D 94(10), 104050 (2016)

66. R. Myrzakulov, L. Sebastiani, S. Vagnozzi, Eur. Phys. J. C 75 , 444 (2015)

67. S.D. Odintsov, V.K. Oikonomou, Astrophys. Space Sci. 361, 236 (2016)

68. S.D. Odintsov, V.K. Oikonomou, Nucl. Phys. B 929, 79 (2018)

69. A.H. Chamseddine, V. Mukhanov, JCAP 1703, 009 (2017)

70. S. Brahma, A. Golovnev, D.-H. Yeom, Phys. Lett. B 782, 280 (2018)

71. J. De Haro, L. A. Saló, S. Pan, Gen. Relativ. Gravit. 51(4), 49 (2019)

72. R. Myrzakulov, L. Sebastiani, S. Vagnozzi, S. Zerbini, Class. Quantum Gravity 33, 125005 (2016)

73. S. Vagnozzi, Class. Quantum Gravity 34, 185006 (2017)

74. R. Myrzakulov, L. Sebastiani, Gen. Relativ. Gravit. 47, 89 (2015)

75. D. Momeni, P.H.R.S. Moraes, H. Gholizade, R. Myrzakulov, Int. J. Geom. Methods Mod. Phys. 15(6), 1850091 (2018)

76. A.V. Astashenok, S.D. Odintsov, Phys. Rev. D 94, 063008 (2016)

77. A.H. Chamseddine, V. Mukhanov, Eur. Phys. J. C 77, 183 (2017)

78. G.G.L. Nashed, Int. J. Geom. Methods Mod. Phys. 15(9), 1850154 (2018) 
79. G.G.L. Nashed, W. El Hanafy, K. Bamba, JCAP 1901(1), 058 (2019)

80. A.H. Chamseddine, V. Mukhanov, T.B. Russ, JHEP 10, 104 (2019)

81. K. Hammer, P. Jirousek, A. Vikman, e-print. arXiv:2001.03169

82. J. Sakstein, B. Jain, Phys. Rev. Lett. 119(25), 251303 (2017)

83. T. Baker, E. Bellini, P.G. Ferreira, M. Lagos, J. Noller, I. Sawicki, Phys. Rev. Lett. 119(25), 251301 (2017)

84. D. Langlois, R. Saito, D. Yamauchi, K. Noui, Phys. Rev. D 97(6), 061501 (2018)

85. R.A. Battye, F. Pace, D. Trinh, Phys. Rev. D 98(2), 023504 (2018)

86. M. Rinaldi, L. Sebastiani, A. Casalino, S. Vagnozzi, Phys. Dark Univ. 22, 108 (2018)

87. A. Ganz, N. Bartolo, P. Karmakar, S. Matarrese, JCAP 1901(01), 056 (2019)

88. A. Casalino, M. Rinaldi, L. Sebastiani, S. Vagnozzi, Class. Quantum Gravity 36(1), 017001 (2019)

89. A. Einstein, The Meaning of Relativity: Fifth edition, including the RELATIVISTIC THEORY OF THE NON-SYMMETRIC FIELD (Princeton University Press, New Jersey, 1970)

90. A. Trautman, Nature 242, 7 (1973)

91. F.W. Hehl, P. Von Der Heyde, G. Kerlick, J. Nester, Rev. Mod. Phys. 48, 393 (1976)

92. A.K. Raychaudhuri, Theoretical Cosmology (Clarendon Press, Oxford, 1979)

93. V. de Sabbata, M. Gasperini, Introduction to Gravitation (World Scientific, Singapore, 1985)

94. V. de Sabbata, C. Sivaram, Spin Torsion and Gravitation (World Scientific, Singapore, 1994)

95. F.W. Hehl, J.D. McCrea, E.W. Mielke, Y. Neéman, Phys. Rep. 258, 1 (1995)

96. F.W. Hehl, Y.N. Obukhov, Lect. Notes Phys. 562, 479 (2001)

97. I.L. Shapiro, Phys. Rep. 357, 113 (2002)

98. M. Blagojevic, Gravitation and Gauge Symmetries (IOP Publishing, London, 2002)

99. L. Fabbri, Higher-Order Theories of Gravitation, Ph.D. Thesis, e-print. arXiv:0806.2610

100. S. Capozziello, M. De Laurentis, Phys. Rep. 509, 167 (2011)

101. N. Poplawski, Gen. Relativ. Gravit. 46, 1625 (2014)

102. H.F. Westman, T.G. Zlosnik, Ann. Phys. 361, 330 (2015)

103. J.A. Musante, Conformal Gauge Relativity: On the Geometrical Unification of Gravitation and Gauge Fields, e-print. arXiv: 1008.2677

104. L. Fabbri, Phys. Lett. B 707, 415 (2012)

105. D.R. Bergman, Internal Symmetry of Space-Time Connections with Torsion, e-print. arXiv: 1411.5568

106. P. Majumdar, S. SenGupta, Class. Quantum Gravity 16, L89 (1999)

107. R.T. Hammond, Gen. Relativ. Gravit. 32, 2007 (2000)

108. B. Mukhopadhyaya, S. Sen, S. SenGupta, Phys. Rev. Lett. 89, 121101 (2002) [Erratum ibid. 89, 259902 (2002)]

109. S. Bhattacharjee, A. Chatterjee, Phys. Rev. D 83, 106007 (2011)

110. B. Mukhopadhyaya, S. Sen, S. SenGupta, Phys. Rev. D 79, 124029 (2009)

111. R. Ferraro, F. Fiorini, Phys. Rev. D 75, 084031 (2007)

112. G.R. Bengochea, R. Ferraro, Phys. Rev. D 79, 124019 (2009)

113. B. Li, T.P. Sotiriou, J.D. Barrow, Phys. Rev. D 83, 104017 (2011)

114. Y.-F. Cai, S.-H. Chen, J.B. Dent, S. Dutta, E.N. Saridakis, Class. Quantum Gravity 28, 215011 (2011)

115. C.G. Böhmer, A. Mussa, N. Tamanini, Class. Quantum Gravity 28, 245020 (2011)

116. Y.-F. Cai, S. Capozziello, M. De Laurentis, E.N. Saridakis, Rep. Prog. Phys. 79, 106901 (2016)

117. S. Bahamonde, C.G. Böhmer, Eur. Phys. J. C 76(10), 578 (2016)

118. S. Bahamonde, S. Capozziello, M. Faizal, R.C. Nunes, Eur. Phys. J. C 77(9), 628 (2017)
119. H.-J. Yo, J.M. Nester, Mod. Phys. Lett. A 22, 2057 (2007)

120. A.V. Minkevich, A.S. Garkun, V.I. Kudin, Class. Quantum Gravity $\mathbf{2 4}, 5835$ (2007)

121. J.M. Nester, L.L. So, T. Vargas, Phys. Rev. D 78, 044035 (2008)

122. P. Baekler, F.W. Hehl, J.M. Nester, Phys. Rev. D 83, 024001 (2011)

123. C.-Q. Geng, C.-C. Lee, H.-H. Tseng, JCAP 1211, 013 (2012)

124. M. Blagojevic, F.W. Hehl, Gauge Theories of Gravitation: A Reader with Commentaries (World Scientific, Singapore, 2013)

125. J. Lu, G. Chee, JHEP 1605, 024 (2016)

126. Y.N. Obukhov, Int. J. Geom. Methods Mod. Phys. 15(suppl. 1), 1840005 (2018)

127. S. Hojman, M. Rosenbaum, M.P. Ryan, Phys. Rev. D 19, 430 (1979)

128. S.M. Carroll, G.B. Field, Phys. Rev. D 50, 3867 (1994)

129. A. Saa, Gen. Relativ. Gravit. 29, 205 (1997)

130. A.S. Belyaev, I.L. Shapiro, Phys. Lett. B 425, 246 (1998)

131. N.J. Poplawski, J. Math. Phys. 47, 112504 (2006)

132. M. Blagojevic, B. Cvetkovic, Phys. Rev. D 88, 104032 (2013)

133. V. Nikiforova, T. Damour, Phys. Rev. D 97(12), 124014 (2018)

134. G. Allemandi, M. Capone, S. Capozziello, M. Francaviglia, Gen. Relativ. Gravit. 38, 33 (2006)

135. V. Faraoni, S. Capozziello, Beyond Einstein Gravity: A Survey of Gravitational Theories for Cosmology and Astrophysics, Fundamental Theories of Physics 170 (2010) (Springer, Dordrecht, 2011)

136. D.A. Carranza, S. Mendoza, L.A. Torres, Eur. Phys. J. C 73, 2282 (2013)

137. F.W. Hehl, YuN Obukhov, G.F. Rubilar, M. Blagojevic, Phys. Lett. A 347, 14 (2005)

138. J.B. Fonseca-Neto, C. Romero, S.P.G. Martinez, Gen. Relativ. Gravit. 45, 1579 (2013)

139. S. Vignolo, L. Fabbri, C. Stornaiolo, Ann. Phys. 524, 826 (2012)

140. J.-A. Lu, Ann. Phys. 354, 424 (2015)

141. L. Fabbri, Int. J. Geom. Methods Mod. Phys. 12(9), 1550099 (2015)

142. T.B. Vasilev, J.A.R. Cembranos, J.G. Valcarcel, P. MartínMoruno, Eur. Phys. J. C 77(11), 755 (2017)

143. R.K. Kaul, S. Sengupta, Phys. Rev. D 93(8), 084026 (2016)

144. R.K. Kaul, S. Sengupta, Phys. Rev. D 94(10), 104047 (2016)

145. S. Sengupta, Phys. Rev. D 96(10), 104031 (2017)

146. R.K. Kaul, S. Sengupta, Phys. Rev. D 96(10), 104011 (2017)

147. S. Sengupta, Phys. Rev. D 97(12), 124038 (2018)

148. S. Sur, A.S. Bhatia, JCAP 1707, 039 (2017)

149. A.S. Bhatia, S. Sur, e-print. arXiv: 1611.06902

150. A.S. Bhatia, S. Sur, Int. J. Mod. Phys. D 26(13), 1750149 (2017)

151. B. Mirza, F. Oboudiat, Gen. Relativ. Gravit. 51(7), 96 (2019)

152. W.-D. Guo, Y. Zhong, K. Yang, T.-T. Sui, Y.-X. Liu, Phys. Lett. B 800, 135099 (2020)

153. F. Izaurieta, P. Medina, N. Merino, P. Salgado, O. Valdivia, JHEP 10, $150(2020)$

154. G.F. Rubilar, Y.N. Obukhov, F.W. Hehl, Class. Quantum Gravity 20, L185 (2003)

155. W.-T. Ni, Phys. Lett. A 378, 1217 (2014)

156. S. SenGupta, S. Sur, Phys. Lett. B 521, 350 (2001)

157. S. Kar, P. Majumdar, S. SenGupta, A. Sinha, Eur. Phys. J. C 23, 357 (2002)

158. S. Kar, P. Majumdar, S. SenGupta, S. Sur, Class. Quantum Gravity 19, 677 (2002)

159. S. Kar, S. SenGupta, S. Sur, Phys. Rev. D 67, 044005 (2003)

160. S. SenGupta, S. Sur, JCAP 0312, 001 (2003)

161. D. Maity, S. SenGupta, S. Sur, Eur. Phys. J. C 42, 453 (2005)

162. S. Sur, S. Das, S. SenGupta, JHEP 0510, 064 (2005)

163. J. Alexandre, N.E. Mavromatos, D. Tanner, Phys. Rev. D 78, 066001 (2008)

164. M. de Cesare, N.E. Mavromatos, S. Sarkar, Eur. Phys. J. C 75, $514(2015)$ 
165. S. SenGupta, S. Sur, Europhys. Lett. 65, 601 (2004)

166. D. Maity, S. SenGupta, Class. Quantum Gravity 21, 3379 (2004)

167. D. Maity, S. SenGupta, S. Sur, Phys. Rev. D 72, 066012 (2005)

168. S. Das, A. Dey, S. SenGupta, Class. Quantum Gravity 23, L67 (2006)

169. A. Das, B. Mukhopadhyaya, S. SenGupta, Phys. Rev. D 90, 107901 (2014)

170. S. Chakraborty, S. SenGupta, Ann. Phys. 367, 258 (2016)

171. S. Chakraborty, S. SenGupta, JCAP 1707(7), 045 (2017)

172. S. Chakraborty, S. SenGupta, JCAP 1805(5), 032 (2018)

173. C.-Q. Geng, C.-C. Lee, E.N. Saridakis, Y.-P. Wu, Phys. Lett. B 704, $384(2011)$

174. C.-Q. Geng, C.-C. Lee, E.N. Saridakis, JCAP 1201, 002 (2012)

175. L. Iorio, E.N. Saridakis, Mon. Not. R. Astron. Soc. 427, 1555 (2012)

176. S. Capozziello, P.A. Gonzalez, E.N. Saridakis, Y. Vasquez, JHEP 1302, 039 (2013)

177. M.A. Skugoreva, E.N. Saridakis, A.V. Toporensky, Phys. Rev. D 91, 044023 (2015)

178. G. Kofinas, E. Papantonopoulos, E.N. Saridakis, Phys. Rev. D 91 , 104034 (2015)

179. S. Capozziello, O. Luongo, R. Pincak, A. Ravanpak, Gen. Relativ. Gravit. 50(5), 53 (2018)

180. G. Farrugia, J. Levi Said, V. Gakis, E.N. Saridakis, Phys. Rev. D 97(12), 124064 (2018)

181. K.-F. Shie, J.M. Nester, H.-J. Yo, Phys. Rev. D 78, 023522 (2008)

182. A.V. Minkevich, Phys. Lett. B 678, 423 (2009)

183. V. Nikiforova, S. Randjbar-Daemi, V. Rubakov, Phys. Rev. D 95(2), 024013 (2017)

184. V.A. Kostelecky, N. Russell, J. Tasson, Phys. Rev. Lett. 100, 111102 (2008)

185. E.E. Flanagan, E. Rosenthal, Phys. Rev. D 75, 124016 (2007)

186. O.V. Babourova, B.N. Frolov, Phys. Rev. D 82, 027503 (2010)

187. F.W. Hehl, Y.N. Obukhov, D. Puetzfeld, Phys. Lett. A 377, 1775 (2013)

188. S. Camera, V.F. Cardone, N. Radicella, Phys. Rev. D 89, 083520 (2014)

189. O. Castillo-Felisola, C. Corral, I. Schmidt, A.R. Zerwekh, Mod. Phys. Lett. A 29, 1450081 (2014)

190. S. Lucat, T. Prokopec, Observing Geometrical Torsion, e-print. arXiv: 1705.00889

191. H. Ramo Chothe, A. Dutta, S. Sur, Int. J. Mod. Phys. D 28(15), 1950174 (2019)

192. L. Mirzagholi, A. Vikman, JCAP 1506, 028 (2015)

193. F. Capela, S. Ramazanov, JCAP 1504, 051 (2015)

194. S. Ramazanov, JCAP 1512, 007 (2015)

195. E. Babichev, S. Ramazanov, Phys. Rev. D 95(2), 024025 (2017)

196. R. Hojman, C. Mukku, W.A. Sayed, Phys. Rev. D 22, 1915 (1980)

197. S. Holst, Phys. Rev. D 53, 5966 (1996)

198. G. Immirzi, Class. Quantum Gravity 14(10), L177 (1997)

199. C. Rovelli, Quantum Gravity (Cambridge University Press, Cambridge, 2004)

200. A. Perez, C. Rovelli, Phys. Rev. D 73, 044013 (2006)

201. T. Thiemann, Modern Canonical Quantum General Relativity (Cambridge University Press, Cambridge, 2007)

202. R.K. Kaul, Phys. Rev. D 77, 045030 (2008)

203. K. Banerjee, Class. Quantum Gravity 27, 135012 (2010)

204. F. Pfaeffle, C.A. Stephan, Commun. Math. Phys. 307, 261 (2011) [Erratum ibid. 313, 291 (2012)]

205. I.L. Shapiro, P.M. Teixeira, Class. Quantum Gravity 31, 185002 (2014)

206. C. Rovelli, F. Vidotto, Covariant Loop Quantum Gravity: An Elementary Introduction to Quantum Gravity and Spinfoam Theory (Cambridge University Press, Cambridge, 2014)

207. A. Ashtekar, Phys. Rev. Lett. 57, 2244 (1986)
208. A. Ashtekar, Phys. Rev. D 36, 1587 (1987)

209. J.F.G. Barbero, Phys. Rev. D 51(10), 5507 (1995)

210. A. Ashtekar, J. Lewandowski, Class. Quantum Gravity 21, R53 (2004)

211. S. Mercuri, Phys. Rev. D 77, 024036 (2008)

212. B. Mukhopadhyaya, S. SenGupta, S. Sur, Mod. Phys. Lett. A 17, 43 (2002)

213. B. Mukhopadhyaya, S. Sen, S. SenGupta, S. Sur, Eur. Phys. J. C 35, 129 (2004)

214. D. Maity, P. Majumdar, S. SenGupta, JCAP 0406, 005 (2004)

215. A. Chatterjee, P. Majumdar, Phys. Rev. D 72, 066013 (2005)

216. L. Freidel, D. Minic, T. Takeuchi, Phys. Rev. D 72, 104002 (2005)

217. S. Mercuri, Phys. Rev. Lett. 103, 081302 (2009)

218. G. Calcagni, S. Mercuri, Phys. Rev. D 79, 084004 (2009)

219. S. Mercuri, V. Taveras, Phys. Rev. D 80, 104007 (2009)

220. F. Bombacigno, F. Cianfrani, G. Montani, Phys. Rev. D 94(6), 064021 (2016)

221. F. Bombacigno, G. Montani, Phys. Rev. D 97, 124066 (2018)

222. F. Bombacigno, G. Montani, Phys. Rev. D 99(6), 064016 (2019)

223. Planck Collaboration, N. Aghanim et al., Planck intermediate results. XLIX. Parity-violation constraints from polarization data, e-print. arXiv: 1605.08633

224. C. Armendariz-Picon, V. Mukhanov, P.J. Steinhardt, Phys. Rev. Lett. 85, 4438 (2000)

225. C. Armendariz-Picon, V. Mukhanov, P.J. Steinhardt, Phys. Rev. D 63, $103510(2001)$

226. M. Malquarti, E.J. Copeland, A.R. Liddle, M. Trodden, Phys. Rev. D 67, 123503 (2003)

227. R.J. Scherrer, Phys. Rev. Lett. 93, 011301 (2004)

228. S. Sur, S. Das, JCAP 0901, 007 (2009)

229. A. Dutta, S. Sur, Mimetic-Metric-Torsion Cosmology from first principles (in preparation)

230. Planck Collaboration, N. Aghanim et. al., Planck 2018 results. VI. Cosmological parameters, e-print. arXiv:1807.06209

231. J. Maldacena, Einstein Gravity from Conformal Gravity, e-print. arXiv: 1105.5632

232. S. Sur, A.S. Bhatia, Class. Quantum Gravity 31, 025020 (2014)

233. S.W. Hawking, G.F.R. Ellis, The Large Scale Structure of SpaceTime (Cambridge University Press, Cambridge, 1973)

234. R.M. Wald, General Relativity (University of Chicago Press, Chicago, 1984)

235. E. Poisson, A Relativist's Toolkit: The Mathematics of Black Hole Mechanics (Cambridge University Press, Cambridge, 2004)

236. S. Sur, A. Dutta, On the emergent dark energy-matter scenarios in Mimetic-Metric-Torsion gravity (in preparation)

237. P. Luz, F.C. Mena, J. Math. Phys. 61(1), 012502 (2020)

238. D. Puetzfeld, Y.N. Obukhov, Phys. Rev. D 76, 084025 (2007) [Erratum ibid Phys. Rev. D 79, 069902 (2009)]

239. M. Chevallier, D. Polarski, Int. J. Mod. Phys. D 10, 213 (2001)

240. E.V. Linder, Phys. Rev. Lett. 90, 091301 (2003)

241. A. Dutta, H. Ramo Chothe, S. Sur, On the aversion of instabilities in Mimetic-Metric-Torsion Cosmology (in preparation)

242. J. Maldacena, JHEP 0305, 013 (2003) 